\title{
Imp2 controls oxidative phosphorylation and is crucial for preserving glioblastoma cancer stem cells
}

\author{
Michalina Janiszewska, ${ }^{1,8}$ Mario L. Suvà,, ${ }^{2,8}$ Nicolo Riggi, ${ }^{2}$ Riekelt H. Houtkooper, ${ }^{3,4}$ \\ Johan Auwerx, ${ }^{3}$ Virginie Clément-Schatlo, ${ }^{5}$ Ivan Radovanovic, ${ }^{5}$ Esther Rheinbay, ${ }^{2,6}$ \\ Paolo Provero, ${ }^{7}$ and Ivan Stamenkovic ${ }^{1,9}$
}

\begin{abstract}
${ }^{1}$ Experimental Pathology, Department of Laboratories, CHUV, University of Lausanne, Lausanne CH-1011, Switzerland; ${ }^{2}$ James Homer Wright Pathology Laboratories, Department of Pathology, Massachusetts General Hospital, Harvard Medical School, Boston, Massachusetts 02114, USA; ${ }^{3}$ Laboratory for Integrative and Systems Physiology, Nestle Chair in Energy Metabolism (NCEM), Ecole Polytechnique Fédérale de Lausanne, Lausanne CH-1015, Switzerland; ${ }^{4}$ Laboratory Genetic Metabolic Diseases, University of Amsterdam, Academic Medical Center, Amsterdam 1105 AZ, Netherlands; ${ }^{5}$ Department of Clinical Neurosciences, University Hospital of Geneva, Geneva CH-1211, Switzerland; ${ }^{6}$ Bioinformatics Program, Boston University, Boston, Massachusetts 02215, USA; ${ }^{7}$ Department of Genetics, Biology, and Biochemistry, University of Torino, Torino 10126 Italy
\end{abstract}

Growth of numerous cancer types is believed to be driven by a subpopulation of poorly differentiated cells, often referred to as cancer stem cells (CSCs), that have the capacity for self-renewal, tumor initiation, and generation of nontumorigenic progeny. Despite their potentially key role in tumor establishment and maintenance, the energy requirements of these cells and the mechanisms that regulate their energy production are unknown. Here, we show that the oncofetal insulin-like growth factor 2 mRNA-binding protein 2 (IMP2, IGF2BP2) regulates oxidative phosphorylation (OXPHOS) in primary glioblastoma (GBM) sphere cultures (gliomaspheres), an established in vitro model for CSC expansion. We demonstrate that IMP2 binds several mRNAs that encode mitochondrial respiratory chain complex subunits and that it interacts with complex I (NADH:ubiquinone oxidoreductase) proteins. Depletion of IMP2 in gliomaspheres decreases their oxygen consumption rate and both complex I and complex IV activity that results in impaired clonogenicity in vitro and tumorigenicity in vivo. Importantly, inhibition of OXPHOS but not of glycolysis abolishes GBM cell clonogenicity. Our observations suggest that gliomaspheres depend on OXPHOS for their energy production and survival and that IMP2 expression provides a key mechanism to ensure OXPHOS maintenance by delivering respiratory chain subunit-encoding mRNAs to mitochondria and contributing to complex I and complex IV assembly.

[Keywords: Imp2; cancer stem cells; OXPHOS; glioblastoma; respiratory complex; mitochondria]

Supplemental material is available for this article.

Received January 26, 2012; revised version accepted July 19, 2012.

A growing number of malignancies are recognized to be composed of phenotypically heterogeneous cells that are hierarchically organized and have diverse degrees of differentiating, proliferative, and tumorigenic capacity (Frank et al. 2010; Clevers 2011; Magee et al. 2012). The apex of the hierarchy is believed to be occupied by undifferentiated cells that can self-renew, give rise to rapidly proliferating cell subpopulations, and reconstitute a phenocopy of the primary tumor upon injection into immunocompromised mice-properties that have earned them

\footnotetext{
${ }^{8}$ These authors contributed equally to the work.

${ }^{9}$ Corresponding author

E-mail ivan.stamenkovic@chuv.ch

Article published online ahead of print. Article and publication date are online at http://www.genesdev.org/cgi/doi/10.1101/gad.188292.112.
}

the denomination of cancer stem cells (CSCs). Despite being presumed to constitute the key cell subpopulation that determines tumor development, CSCs are functionally defined on the basis of no more than a handful of properties, chief among which are their ability to initiate tumor growth and give rise to all tumor cell subpopulations in vivo as assessed by xenotransplantation assays. Resistance to therapy is often attributed to CSCs but does not constitute a defining trait (Dean et al. 2005). A major challenge to the isolation of CSCs from solid tumors is the paucity of reliable means to identify them. Whereas the cell surface receptors CD34 and CD38 have helped define leukemia stem cells based on their convincing validation as markers of normal hematopoietic stem cells (Lapidot et al. 1994), the developmental hierarchy of most tissues that develop solid tumors is insufficiently char- 
acterized to provide a robust basis for CSC identification. Several single cell surface receptors, including CD133, CD44, and SSEA, or combinations thereof, have been used to identify CSCs in glioblastoma (GBM) based on their expression in subpopulations of cells with selfrenewal and tumor-initiating properties (Singh et al. 2004; Son et al. 2009; Anido et al. 2010). These receptors have proved to be valid markers of CSCs in freshly removed tumors, but it remains critical to complement any marker used by stringent functional experiments to identify cells with high tumor-initiating potential whose study is essential for better understanding of tumor development.

Single cells with self-renewing and tumor-initiating capacity from a variety of solid tumors are able to form spheres upon in vitro culture under defined serum-free conditions (Galli et al. 2004; Ricci-Vitiani et al. 2007). GBM spheres (gliomaspheres) have been shown to allow long-term expansion of cells with clonogenic and highly tumorigenic properties that recapitulate the morphology and genetic mutations of the parental tumor (Lee et al. 2006; Wakimoto et al. 2012). They therefore appear to be enriched in cells that correspond to the current functional definition of CSCs and represent a reproducible functional model to address CSC properties.

Because CSCs may, in some cases, display plasticity that is typically associated with developing tissues, oncofetal proteins may participate in and potentially even determine many of their phenotypic and functional features. The oncofetal protein Imp2 (insulin-like growth factor 2 mRNA-binding protein 2) is expressed in the developing mammalian brain, suggesting possible implication in normal embryonic development (Christiansen et al. 2009). Imp2 belongs to the Imp family of proteins that regulate subcellular mRNA localization, translation, and stability (Christiansen et al. 2009) and consists of three pairs of RNA-binding domains: RRM1-2, KH1-2, and KH3-4. Studies on paralog proteins IMP1 and IMP3 have shown a correlation between their expression and poor outcome in various malignancies, including melanoma, ovarian cancer, and pancreatic, prostate, thyroid, breast, and colon carcinoma (Yaniv and Yisraeli 2002; Himoto et al. 2005a,b; Dimitriadis et al. 2007; Kobel et al. 2007), but mechanisms that underlie their functional implication in tumor biology have not been identified so far. Recent evidence suggests that Imp2 may be implicated in type 2 diabetes (Saxena et al. 2007; Scott et al. 2007; Christiansen et al. 2009) and in regulating smooth muscle cell adhesion and motility (Boudoukha et al. 2010), but with the exception of its expression in diverse malignancies (Hammer et al. 2005; Himoto et al. 2005b), virtually nothing is known about its role in cancer. Because of its expression in the developing brain, we chose to address its possible functional role in GBM.

GBM (grade IV astrocytoma) is among the most malignant forms of brain tumor, with notorious resistance to conventional anti-cancer therapy. On average, patients with GBM survive for no more than 1 year following diagnosis (Louis 2006). Cells that fulfill the currently accepted functional CSC criteria have been isolated from
GBM based on their expression of diverse cell surface markers, including CD133 (Singh et al. 2004), SSEA1 (Son et al. 2009), and CD44 (Anido et al. 2010). Moreover, single self-renewing GBM cells form gliomaspheres in culture that display properties consistent with CSC enrichment (Lee et al. 2006; Wakimoto et al. 2009, 2012). GBM thus provides a suitable solid tumor model to investigate CSC properties that distinguish them from cells that comprise the tumor bulk. We therefore addressed Imp2 expression, the repertoire of its target mRNAs, and its functional relevance in GBM. Our observations show that Imp2 is expressed in GBM and that its expression is highest in $\mathrm{CD} 33^{+}$cells from freshly isolated tumors, in gliomasphere cultures in vitro, and in cells associated with blood vessels and necrotic areas in vivo, where CSCs are believed to reside. We also demonstrate that Imp2 binds mitochondrial respiratory complex IV (CIV) mRNAs and complex I (CI) proteins and participates in their assembly and function, thereby regulating oxidative phosphorylation (OXPHOS). Remarkably, Imp2 expression is shown not only to maintain OXPHOS in tumor cells, but to be required for the survival and function of cells that display self-renewal and tumor-initiating properties. Our observations define a new function for Imp2 that may constitute a therapeutic target in one of the most malignant human tumors.

\section{Results}

Imp2 expression in normal brain and glial tumors

Immunohistochemical staining of a normal adult brain and a panel of gliomas of varying grades revealed that Imp2 is absent in normal brains and grade II and III astrocytomas (Fig. 1A), whereas 40 out of 51 GBM samples were Imp2positive. Correlation between Imp2 expression and glioma grading was confirmed by microarray analysis of Imp2 expression in an independent data set of 153 gliomas of different grades and 23 normal samples (Fig. 1B; Sun et al. 2006). Some variability in Imp2 expression is observed among GBM subtypes, as defined according to The Cancer Genome Atlas (TCGA) database (http://cancergenome. nih.gov), with the lowest levels found in the neural subtype (Fig. 1C). Interestingly, elevated Imp2 expression correlates with poor prognosis in the proneural GBM subtype (Fig. 1D), the molecular subgroup that is the most refractory to current therapy (Verhaak et al. 2010). No prognostic significance of Imp2 was observed in other GBM subtypes (data not shown).

Closer examination of GBM tissue sections revealed that Imp2 is weakly expressed throughout the tumors, but that cells that display the strongest reactivity to antiImp2 antibody localize primarily around blood vessels and hypoxic areas bordering tumor necrosis (Fig. 1A). Both locations have been shown to be enriched in CSCs (Li et al. 2009; Anido et al. 2010; Charles et al. 2010). To determine Imp2 expression in cells that correspond to GBM CSCs, we performed quantitative real-time PCR (qRT-PCR) analysis of RNA extracted from freshly sorted primary GBM cells that had not been subjected to in vitro culture. We used CD133 expression to identify cells with 
A

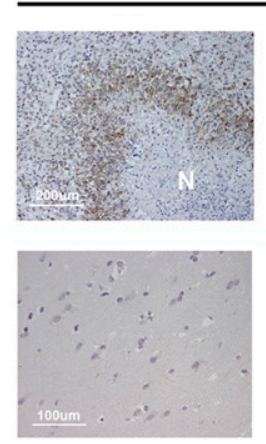

Normal brain
Glioblastoma (grade IV)
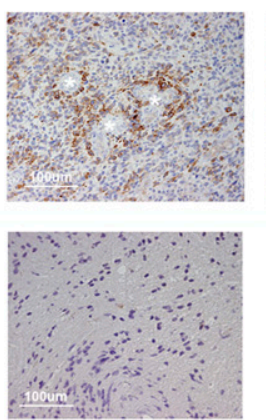

grade II
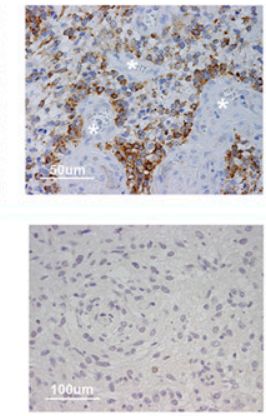

grade III

Astrocytoma
D

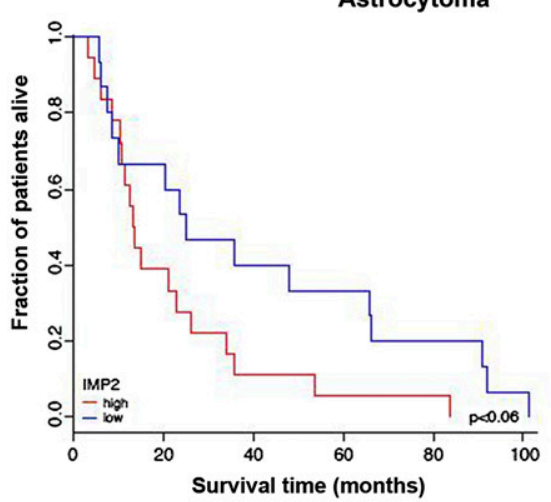

B

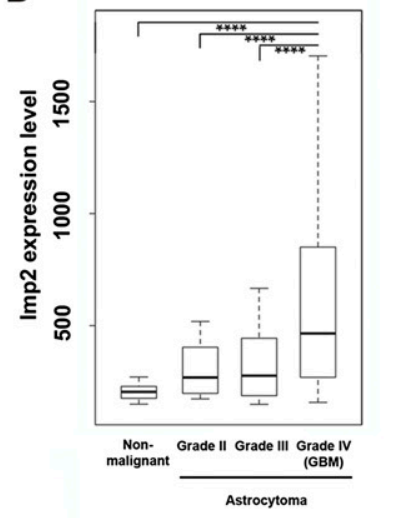

C

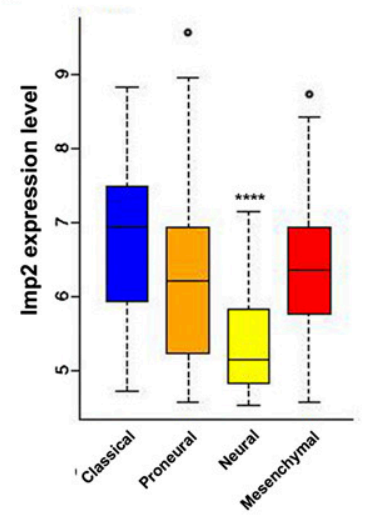

$\mathbf{E}$

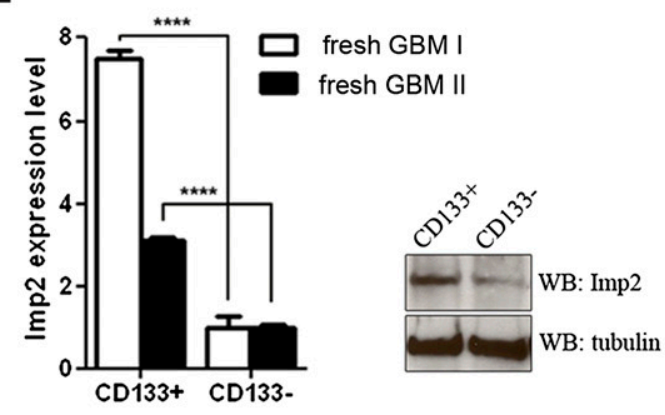

G
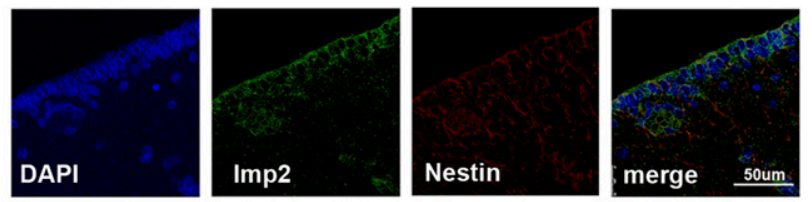

Figure 1. Imp2 expression in GBM. (A, top panel) Imp2-positive cells in GBM localize predominantly to perinecrotic areas $(\mathrm{N})$ and in the vicinity of blood vessels (denoted by asterisks). (Bottom panel) Immunohistochemical staining of paraffin GBM sections shows the absence of Imp2 expression in normal brains and grade II and III astrocytomas. (B) Imp2 expression in astrocytomas of different grades in an independent data set (Sun et al. 2006); $t$-test $P$-values: GBM versus normal brain: $P=9.678 \times 10^{-11}$; GBM versus grade II/III astrocytoma: $P=4.310 \times 10^{-5}$. Whiskers represent the 95th percentile. (C) IMP2 expression differs significantly among TCGA molecular subtypes (Kruskal-Wallis test $P$-value $<10^{-7}$ ) (Verhaak et al. 2010). (D) Kaplan-Meier survival curves for proneural TCGA samples with "high" or "low" IMP2 expression (see the Materials and Methods). Median survival of the "Imp2 high" group is 13.3 mo compared with 24.9 for patients in the "Imp2 low" group; log-rank test $P$-value $<0.06$. (E) Imp2 expression in GBM CD133 ${ }^{+}$cells. (Left panel) Cells from two surgical biopsies of GBM patients were sorted for CD133 expression. Imp2 transcripts as measured by qRT-PCR were significantly higher in $\mathrm{CD}_{133^{+}}$populations. $\left(^{\star \star \star \star}\right) P<0.0001$ (unpaired two-tailed $t$-test). (Right panel) Higher level of Imp2 protein expression in freshly isolated CD133+ cells was confirmed by Western blot analysis. (F) Imp2 expression in GBM CSCs in situ. Double immunofluorescence staining shows that GBM cells expressing nestin are also positive for Imp2. $(G)$ Costaining of Imp2 and stem/progenitor cell marker nestin in the SVZ of fetal brains.

CSC features, given that CD133 has been shown to be a reliable marker of freshly removed self-renewing and tumorigenic GBM cells (Singh et al. 2004; Bao et al. 2006). Threefold higher Imp transcript expression was observed in $\mathrm{CD}_{133^{+}}$than in $\mathrm{CD} 133^{-}$populations (Fig. 1E, left), and expression of Imp2 protein was correspondingly higher in $\mathrm{CD}_{133^{+}}$cells, as assessed by Western blot analysis using an anti-Imp2 antibody (Fig. 1E, right). Imp2 was also shown to be expressed in GBM cells bearing the stem/progenitor cell markers SSEA1 (Supplemental Fig. 1A) and nestin (Fig. $1 \mathrm{~F})$ by immunofluorescence costaining in situ.
To determine whether Imp2 is present in normal tissue stem cells, Imp2 expression in nestin- and GFAP-positive neural progenitors in a 20th-week human fetal brain was assessed by immunohistochemistry. These cells are localized in the subventricular zone (SVZ) and were found to express Imp2 (Fig. 1G; Supplemental Fig. 1B). Importantly, Imp2 expression, as assessed by immunohistochemistry, was not detected in other areas of the brain (Supplemental Fig. 1C), suggesting a functional role that, in the CNS, may be restricted to stem cells. Imp2 expression was also assessed by Western blot analysis in the 
validated neural progenitor cell line hNP1 and shown to be comparable with that in spherogenic GBM cells (Supplemental Fig. 1D).

To further investigate Imp2 expression in GBM cell subpopulations, we established primary tumor culture conditions that favor CSC maintenance (Galli et al. 2004; Clement et al. 2007). Cells grown under these conditions displayed both self-renewal and sphere-forming capacity in vitro, as well as tumorigenicity in vivo, consistent with CSC enrichment as defined by current criteria. Exposure of the spheres to serum resulted in the cells becoming adherent (Fig. 2A). Flow cytometry analysis revealed that $70 \%$ of sphere-forming cells but $<1 \%$ of adherent cells were $\mathrm{CD} 133^{+}$(Fig. 2B, top panels). Similarly, 43\% of sphere-forming cells, as opposed to $0.19 \%$ of adherent cells, expressed SSEA1 (Fig. 2B, bottom panels).

Spheres and adherent human GBM cells from three independent tumors (BT1-3) were compared for tumorigenicity upon orthotopic injection into immunocompromised mice. Although injection of 50 or 100 spherogenic cells was not sufficient to initiate tumor growth, as few as 500 sphere-derived cells formed tumors that led to the death of $100 \%$ of the animals within $40-60 \mathrm{~d}$. In contrast, none of the animals injected with as many as 100,000 adherent cells developed tumors (Fig. 2C; data not shown). These results are consistent with previous work that compared the tumor-initiating potential of matched patient-derived GBM cells grown in spherogenic and
A
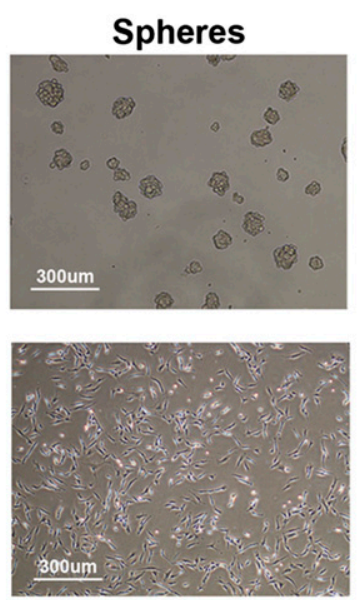

Adherent

C

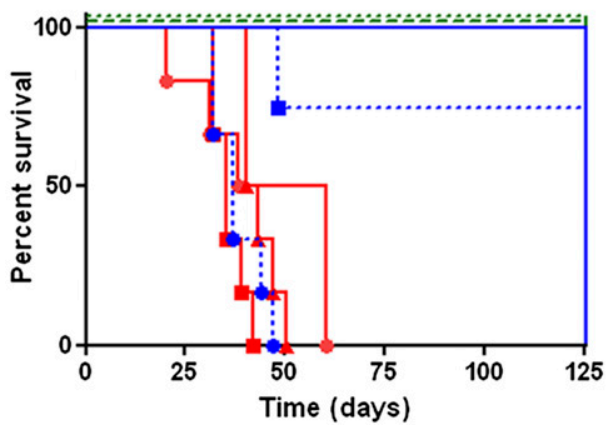

B
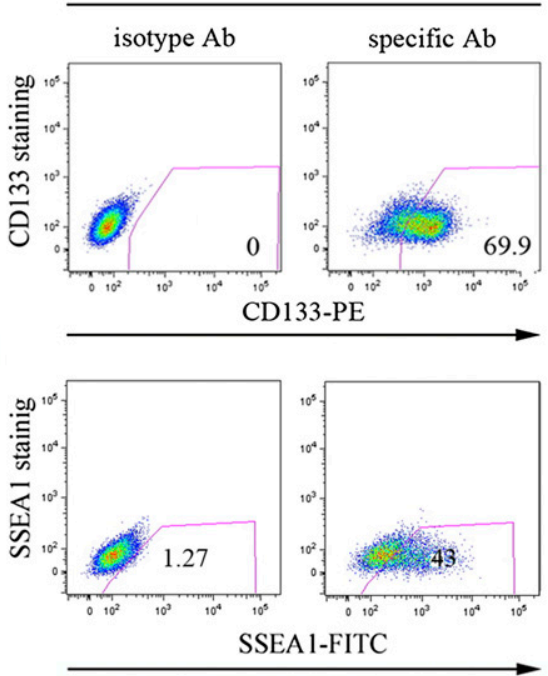

D

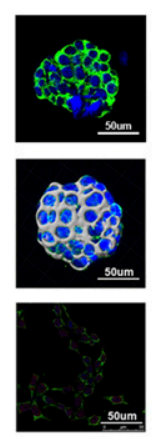

adherent

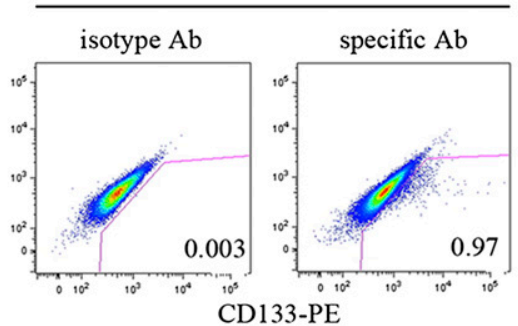

CD133-PE

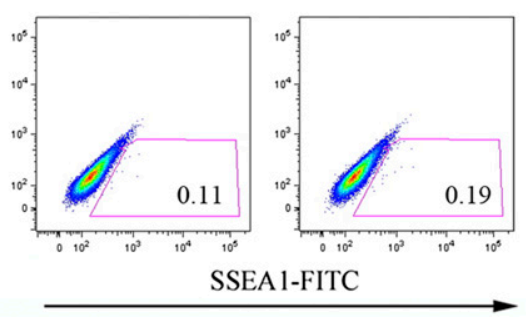

E

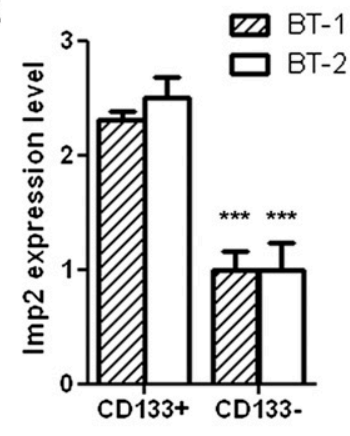

Figure 2. Gliomaspheres as a model of GBM CSC-enriched populations. (A) Morphology of sphere-forming and adherent primary GBM cultures. (B) FACS analysis of CSC-associated marker expression in the two types of primary GBM cultures. Only GBM cultured as spheres express CD133 (top panel) and SSEA1 (bottom panel). Representative results for one out of three GBM samples are shown. (C) Survival of mice after intracranial injection of GBM cells cultured as spheres and adherent cells. Five-hundred spherogenic cells (three independent cell batches, BT1-3) were sufficient to initiate tumor growth, whereas 100,000 adherent cells derived from spheres exposed to serum (BT-1adh) failed to form tumors (data for BT2-3adh are not shown); injection of 50 or 100 spherogenic cells was insufficient for tumor growth initiation (data for BT2-3 are not shown); six animals per condition were injected. $(D)$ Immunofluorescence staining of a GBM spheroid (nonreconstituted image [top panel] and three-dimensional [3D] reconstruction [middle panel]) compared with adherent GBM cells (bottom panel) shows high Imp2 expression levels in a CSC-enriched sphere. (Green immunofluorescence) Imp2 staining; (white) Imp2 channel 3D reconstruction (signal above threshold); (blue) DAPI staining. (E) Imp2 expression in gliomasphere cells sorted for CD133 expression. Imp2 transcripts as measured by qRT-PCR were significantly higher in $\mathrm{CD} 133^{+}$populations. $\left(^{\star \star \star}\right) P=0.0003$ and 0.001 (unpaired two-tailed $t$-test). 
adherent conditions (Lee et al. 2006; Wakimoto et al. 2009, 2012). Tumors formed by spherogenic cells were a phenocopy of the primary malignancies they were derived from (data not shown), which, together with CD133 and SSEA expression, supports the notion that spheres are enriched in CSC-like cells (Suvà et al. 2009).

Elevated Imp2 expression was observed in gliomaspheres, as assessed by immunofluorescence (Fig. 2D, top and middle panels), although anti-Imp2 antibody staining, albeit less intense, was noted in adherent cells as well (Fig. 2D, bottom panel). Quantification by qRT-PCR confirmed twofold to threefold higher Imp2 expression in gliomaspheres than in adherent cells (Supplemental Fig. 1E). Moreover, CD133 ${ }^{+}$GBM sphere-derived cells maintained twofold to threefold higher Imp2 levels than their CD133- counterparts (Fig. 2E). Imp2 expression is therefore more elevated in CSC-enriched GBM cell populations but is not restricted to them.

\section{Imp2 is crucial for GBM CSC maintenance in vitro and in vivo}

To assess the functional relevance of Imp2 expression in GBM, stable repression of Imp2 transcripts using two distinct specific shRNA sequences was achieved in primary GBM sphere-derived cells (Fig. 3A). Cells depleted of Imp2 had markedly impaired spherogenic capacity in culture (Fig. 3B), and the spheres that were formed had a smaller diameter than their wild-type counterparts (Fig. $3 \mathrm{C})$, raising the possibility that both viability and proliferation might be affected. Decreased cell viability, as measured by fluorescence-activated cell sorting (FACS) analysis of Calcein AM-stained cells, was indeed observed (Fig. 3D), as was impaired proliferation (Fig. 3E). Inhibition of sphere-forming capacity by Imp2-depleted cells was verified in clonogenic assays on three independent cell batches where single cells were seeded individually in three 96-well plates per condition and the number of resulting spheres was scored (Fig. 3F; Supplemental Fig. 2A). Propidium iodide staining indicated that Imp2 depletion does not affect the cell cycle but diminishes the number of cycling cells by increasing cell death (Supplemental Fig. 2B). To determine whether the increase in cell death is due to apoptosis, we assessed PARP cleavage, caspase- 3 activation, and annexin $\mathrm{V}$ in Imp2-depleted cells. Imp2 knockdown did not enhance PARP cleavage and activation of caspase 3 , as assessed by Western blot analysis (Supplemental Fig. 2C), and no difference in anti-annexin $\mathrm{V}$ antibody staining was observed between Imp2-expressing and Imp2-depleted cells (data not shown), indicating that Imp2 depletion does not induce apoptosis in gliomaspheres. To verify that GBM sphere-forming cells are depleted upon Imp2 knockdown, we assessed expression of CD133 as well as that of the stemness genes SOX2, OCT4, and NANOG in viable cells bearing Imp2 shRNA. A robust decrease in all four transcripts was observed (Fig. 3G).

We next assessed the effect of Imp2 on the tumorigenic potential in vivo of GBM cells by orthotopic injection of gliomaspheres, depleted or not depleted of Imp2, into the brain of nonobese diabetic-severe combined immunodeficient (NOD/SCID) mice. Animals receiving spherederived cells depleted of Imp2 survived for nearly twice as long as mice injected with sphere-derived cells with unaltered Imp2 levels (Fig. 3H). Immunohistochemical analysis revealed that the tumors that eventually emerged in mice that had received Imp2-depleted cells expressed Imp2 at a level comparable with that of control cellderived counterparts (Supplemental Fig. 2D), indicating that tumor formation results from escapers of $\operatorname{Imp} 2$ depletion. These results provide evidence that Imp2 is required for gliomasphere maintenance and that the tumorigenic potential of gliomaspheres depends on Imp2 expression.

\section{Imp2-bound transcripts and proteins regulate mitochondrial function}

To begin to address the putative role of Imp2 in GBM CSCs, gliomaspheres were used to immunoprecipitate Imp2 ribonucleoprotein complexes and analyze Imp2bound mRNAs on Affymetrix microarrays (RIP [RNA immunoprecipitation]-ChIP [chromatin immunoprecipitation] analysis). Among 400 transcripts that were bound by Imp2, a significant overrepresentation of genes implicated in mitochondrial function and OXPHOS was found (Fig. 4A,B; Supplemental Table 1). Enrichment of a panel of the transcripts chosen based on their association with mitochondrial function in the Imp2-bound fraction was validated by qRT-PCR (Supplemental Fig. 3A). Binding of the selected transcripts to Imp2 was also elevated in the neural progenitor cell line hNP1. Interestingly, their enrichment in the Imp2 RIP fraction from hNP1 cells was nearly a magnitude higher than in the corresponding RIP fraction from gliomaspheres (Supplemental Fig. 3B). To further confirm the specificity of mRNA binding by Imp2, a proximity ligation assay (PLA) was performed. GBM cells were transfected with a biotin-labeled RNA oligomer of the candidate Imp2 target COX7b or of VSV as a control. Anti-Imp2 and anti-biotin antibodies could generate a PLA signal only when they recognize substrates whose mutual proximity corresponds to physical interaction. The signal generated by each pair of labeled anti-biotin and anti-Imp2 antibodies is visualized as a single fluorescent spot (Supplemental Fig. 3C, green). The results clearly indicate binding of COX $7 b$ oligo-RNA by Imp2. Because Imp2 has not been associated with mitochondria thus far and because an adequate energy supply is crucial to tumor cell growth, we addressed the possible implication of Imp2 in mitochondrial metabolism. Depletion of Imp2 decreased the expression of a panel of Imp2-bound mRNAs that encode mitochondrial proteins, which supports functional significance of their binding to Imp2 (Fig. 4C).

In parallel to the assessment of mRNAs bound to Imp2, we conducted pull-down experiments using an anti-Imp2 antibody to identify putative Imp2-interacting proteins. Mass spectrometric detection of several subunits of CI of the mitochondrial respiratory chain in the pull-down material not only strengthened the evidence that Imp2 


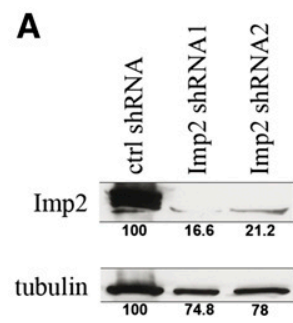

D
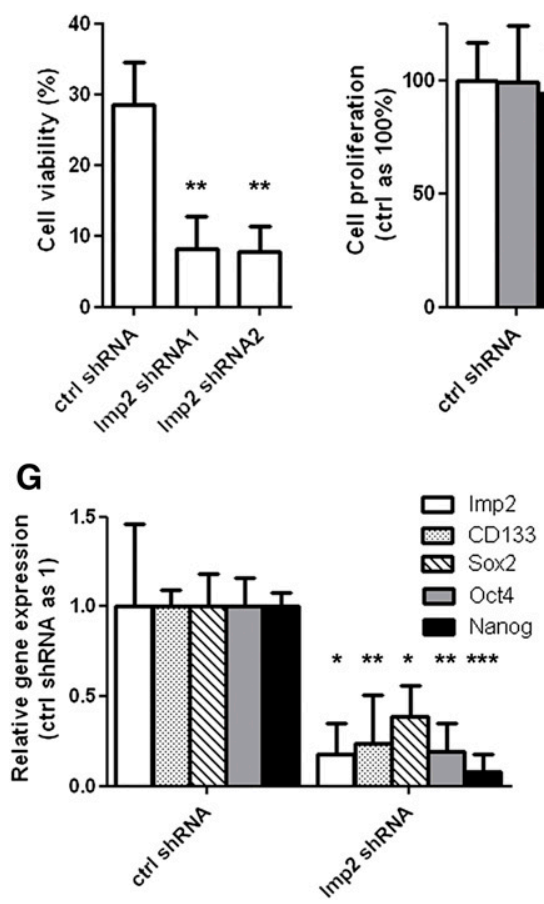

B

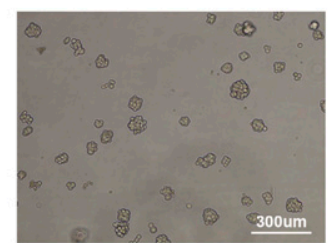

E

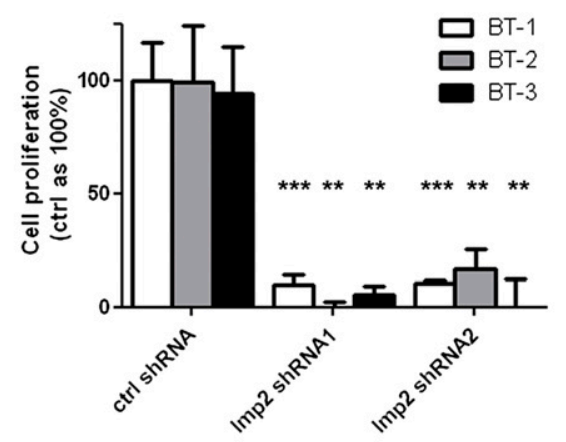

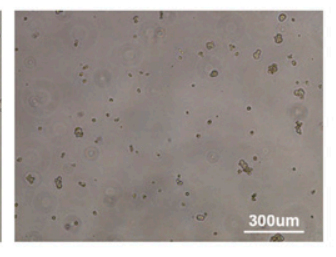
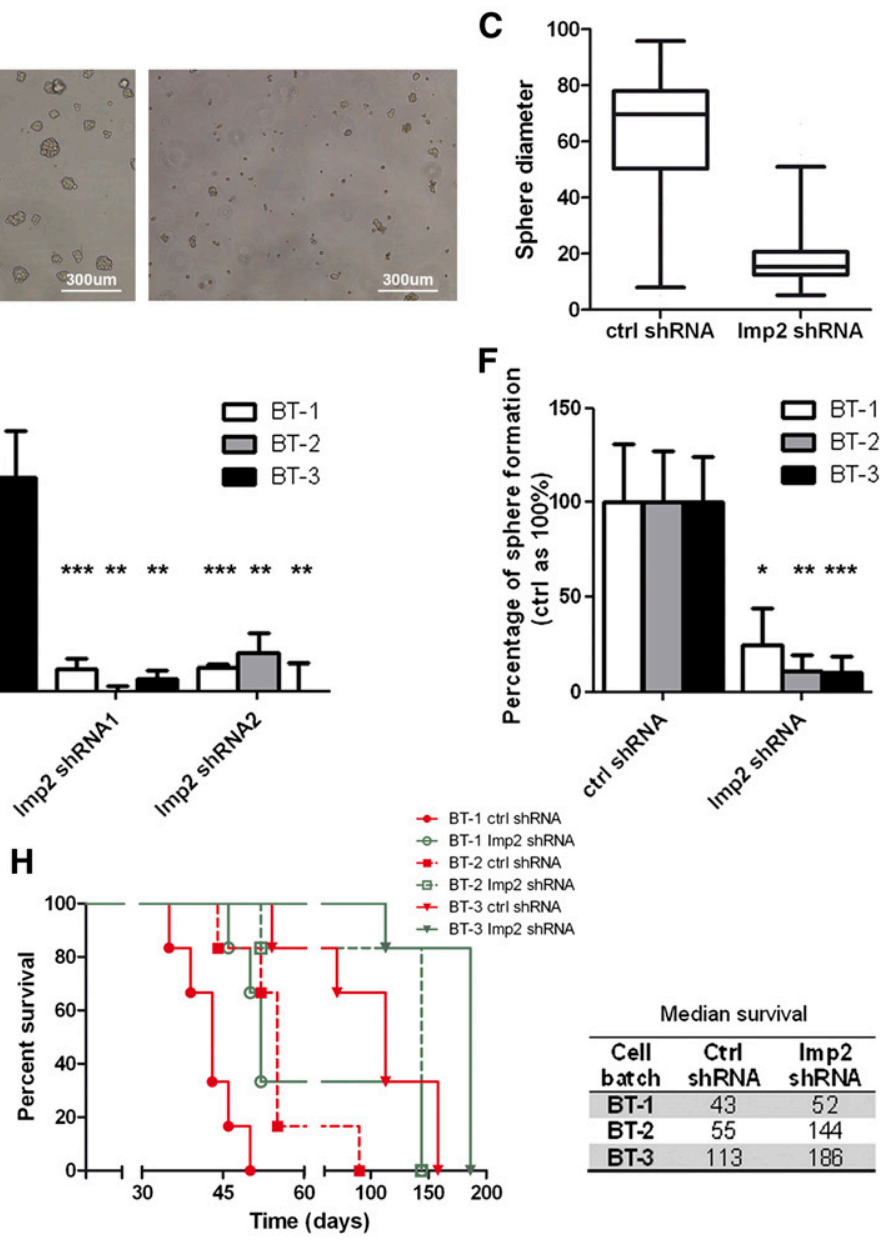

$F_{5}$

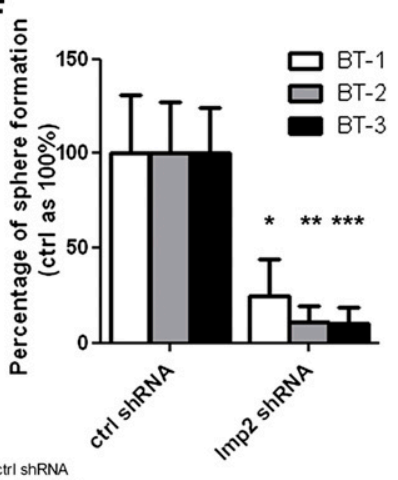

T-1 ctrl ShRNA

BT-1 Imp2 shRNA

BT-2 ctrl shRNA

BT-2 Imp2 shRNA

BT-3 ctrl ShRNA

$$
\text { . }
$$

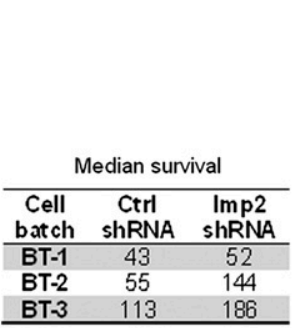

Figure 3. Effect of Imp2 depletion on gliomasphere cells. (A) Imp2 silencing in gliomasphere cells stably expressing two different shRNAs. (B) Morphology of spheres formed by control shRNA-expressing and Imp2-depleted GBM cells in culture. $(C)$ Sphere morphology change upon Imp2 depletion shown as sphere diameter. $P<0.0001(n=50)$, unpaired two-tailed $t$-test. Whiskers represent the 95th percentile. $(D)$ Cell viability as measured by FACS analysis of Calcein AM staining in three different cell batches. Bars represent the percentage of positively stained cells. Low viability of ctrl cells is due to puromycin selection. $\left(^{\star \star}\right) P=0.0094$ and 0.0068 , respectively; paired two-tailed $t$-test. Error bars represent the standard deviation (SD). (E) Cell proliferation in spherogenic culture conditions upon Imp2 knockdown as assessed by BrdU incorporation. ${\left({ }^{\star \star \star}\right)}^{*} P=0.0008$ and $\left.0.0007 ;{ }^{\star \star}\right) P=0.0024,0.0057,0.002$, and 0.0027, respectively; unpaired two-tailed $t$-test. Error bars represent the SD. $(F)$ Single-cell clonogenic assay performed on three independent cell batches; single cells were plated in 96-well plates, and three plates per condition were used; statistical analysis for each cell batch was performed using unpaired two-tailed $t$-test comparing control cells with Imp2-depleted cells. $\left(^{\star}\right) P=0.0227 ;\left(^{\star \star}\right) P=$ $\left.0.0015 ;\left.\right|^{\star \star \star}\right) P=0.0008$; error bars represent the SD. $(G)$ Imp2 depletion decreases CD133, SOX2, OCT4, and NANOG transcripts as measured by qRT-PCR. $\left(^{\star}\right) P=0.0443 ;\left(^{\star \star}\right) P=0.0098 ;\left(^{\star}\right) P=0.013 ;\left(^{\star \star}\right) P=0.0034 ;\left(^{\star \star \star}\right) P=0.0002$. Representative values from two independent experiments are shown. $(H)$ Imp2 shRNA expression decreases GBM CSC tumor-forming capacity. The experiment was repeated using three batches of cells. Six animals were used per condition. The differences between Imp2- and ctrl shRNA-expressing cells for each cell batch were significant (log-rank [Mantel-Cox] test). P-values for BT-1, BT-2, and BT-3 were 0.0088, 0.0036, and 0.0056, respectively. The median survival of the animals is presented in the right panel.

is functionally related to OXPHOS, but suggested at least two distinct mechanistic links (Fig. 4D). CI catalyzes electron transfer from NADH to ubiquinone and constitutes the main entry point of electrons into the respiratory chain (Carroll et al. 2003, 2006; Murray et al. 2003). Two subunits, NADH dehydrogenase (ubiquinone) iron-sulfur protein 3 (NDUFS3) and iron-sulfur protein 7 (NDUFS7), which form part of the peripheral arm of the complex (Fernandez-Vizarra et al. 2009), as well as
NADH dehydrogenase (ubiquinone) $1 \alpha$ complex assembly factor 3 (NDUF3), were identified in the Imp2 pulldown. The interaction between Imp2 and NDUFS3 was verified by immunoprecipitation and Western blot analysis (Fig. 4E). Moreover, protein expression levels of CI subunits identified in the pull-down experiment decreased upon Imp2 knockdown (Fig. 4F,G), supporting the functional relevance of Imp2-CI subunit interactions. 


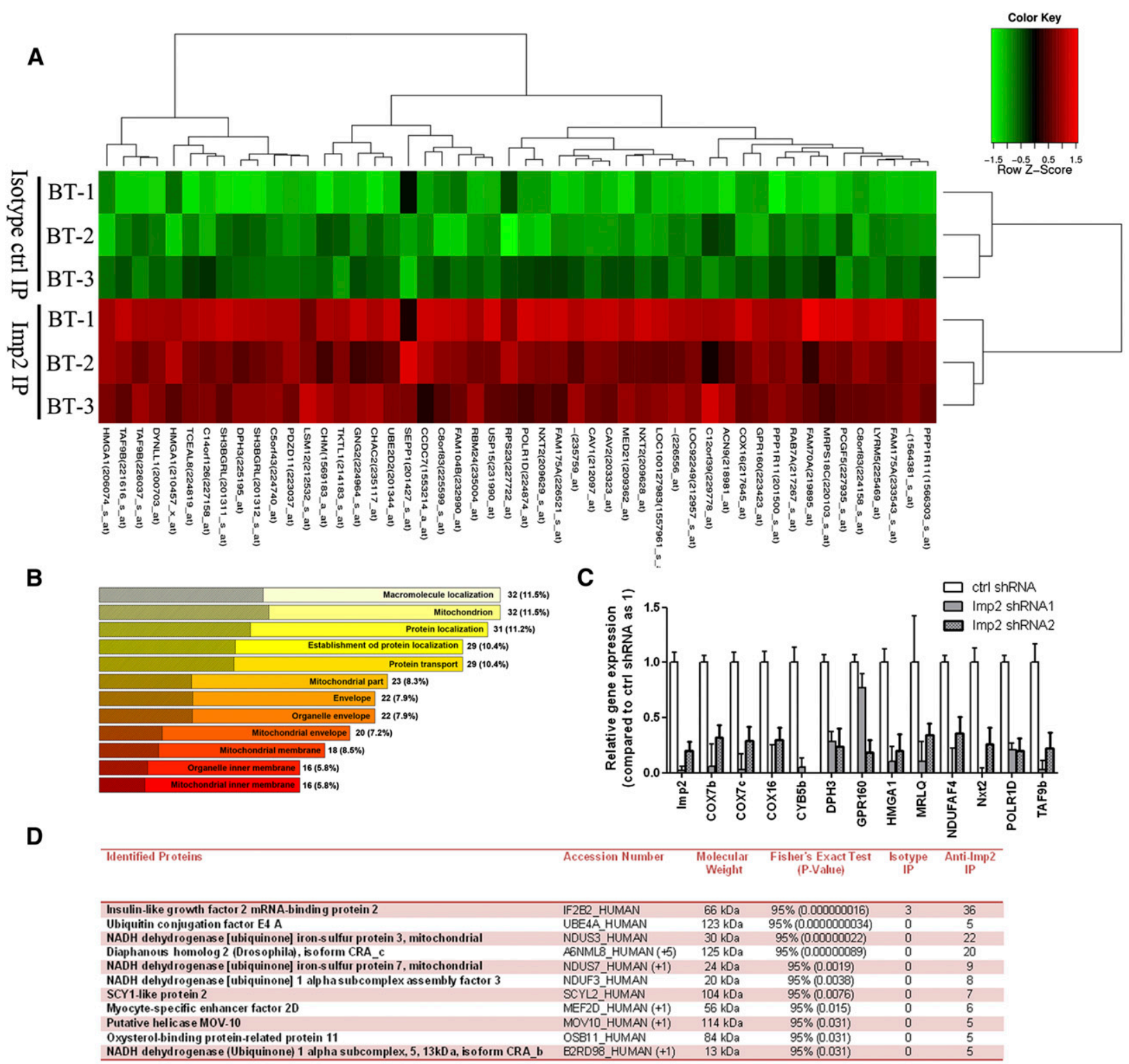

E

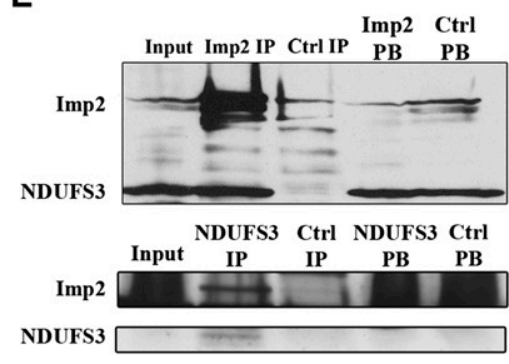

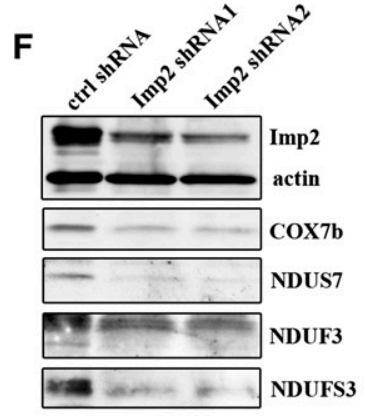

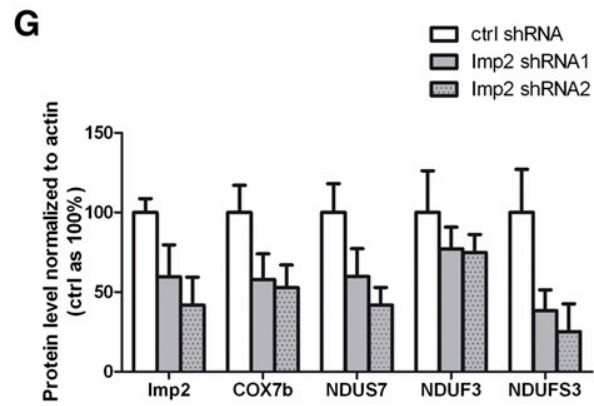

Figure 4. mRNA and proteins bound by Imp2 suggest a functional role in mitochondrial processes. $(A)$ Specificity of the RIP assay is illustrated in a heat map that compares transcripts obtained by immunoprecipitation using anti-Imp2 and isotype-matched control antibody; results for three batches of primary GBM cultures in spherogenic conditions are shown. (B) Gene ontology (GO) analysis of Imp2-bound mRNA shows enrichment in transcripts associated with mitochondrial function. A GO term was considered overrepresented if the nominal $P$ of the exact one-tailed Fisher test was $<10^{-5}$. The length of the bar for each term is proportional to the number of bound mRNAs annotated to the term. The shaded part of the bar represents the number of annotated mRNAs expected by chance. $(C)$ Imp2 knockdown decreases expression of Imp2-bound transcripts (transcripts associated with mitochondrial functions are shown). (D) Identification of several mitochondrial respiratory CI proteins by mass spectrometry analysis of anti-Imp2 antibody pulldown material. $(E)$ Immunoprecipitation of Imp2 followed by Western blotting using an anti-NDUFS3 antibody (top panel) and immunoprecipitation of NDUFS3 followed by Western blotting using an anti-Imp2 antibody (bottom panel) confirm the interaction between the two proteins. (Ctrl) Immunoprecipitation with isotype-matched control antibody; (PB) post-beads unbound fraction. (F) Imp2 depletion decreases the level of Imp2-bound proteins. $(G)$ Densitometric quantification of the Imp2-bound proteins observed in $F$. Data were normalized to actin. 


\section{Depletion of Imp2 impairs OXPHOS in gliomaspheres}

Because Imp2-bound transcripts and proteins are required for OXPHOS, we investigated the effect of Imp2 depletion on the mitochondrial activity of spherogenic GBM cells. MitoTracker Red staining, which provides an indication of mitochondrial activity, was significantly decreased in cells depleted of Imp2 using two distinct shRNA sequences (Fig. 5A). MitoTracker Red staining was performed on viable cells following the exclusion of dead cells by DAPI and Calcein AM staining such that the data reflect the decreased mitochondrial activity in live cells. To address possible changes in mitochondrial activity following Imp2 depletion, oxygen consumption rate (OCR) and extracellular acidification rate (ECAR) measurements that reflect OXPHOS and glycolysis, respectively, were made. These measurements were performed on equal numbers of viable cells from all three populations immediately after cell counting, thereby excluding cell death as an explanation for decreased metabolic activity. Cells expressing either Imp2 shRNA displayed a significantly decreased OCR (Fig. 5B) but an unaltered ECAR (data not shown). Normalization for equal viable cell seeding was performed by collecting cells following oxygen consumption measurement and staining them with Calcein AM. The assay was subsequently performed on cells sorted for $\mathrm{CD}_{133^{+}}$expression from freshly removed GBM, unexposed to in vitro culture, and revealed augmented OCR in comparison with their $\mathrm{CD} 133^{-}$counterparts, suggesting that CSC marker-bearing subpopulations maintain elevated OXPHOS (Fig. 5C). To determine whether the changes in the OCR triggered by Imp2 depletion might reflect an overall decrease in mitochondrial function, enzymatic activity of the tricarboxylic acid cycle enzyme citrate synthase was assessed (Fig. 5D), but no significant decrease in its activity was observed. Decreased OCR in the face of maintained mitochondrial enzymatic activity supports the notion that the effect of Imp2 is selective for OXPHOS. Because all of the observations thus far suggest Imp2 involvement in the regulation of energy production, cellular ATP content was measured. A significant decrease in ATP was observed in viable sphere-derived cells devoid of Imp2 (Fig. 5E).

Our observations that Imp2 binds CI proteins and CIV subunit transcripts and that Imp2 depletion affects both CI protein and CIV mRNA/protein levels suggest that the effects of Imp2 depletion may be related to changes in CI and CIV activity. To address this possibility, we performed
A

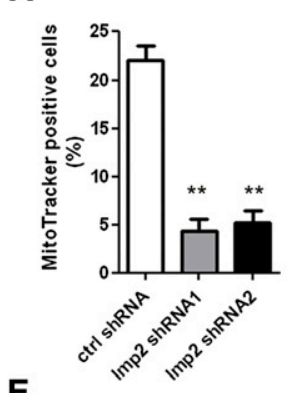

E

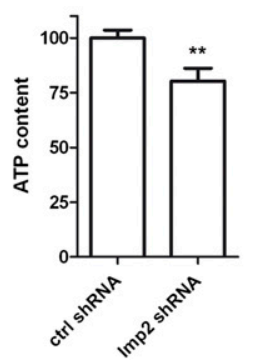

B

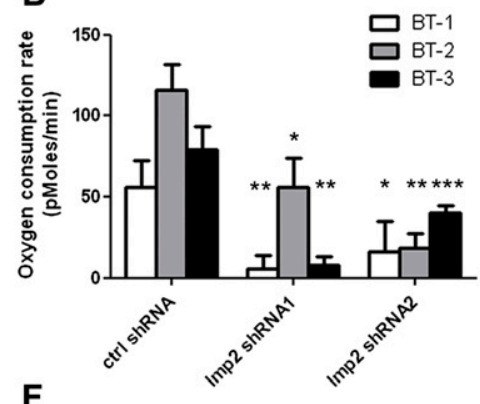

$\mathbf{F}$

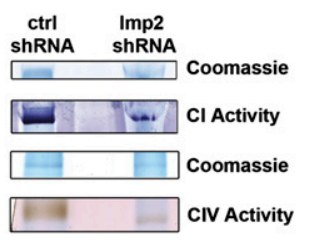

C

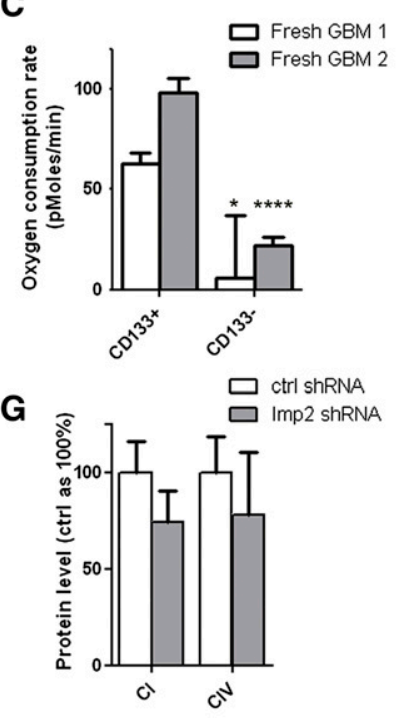

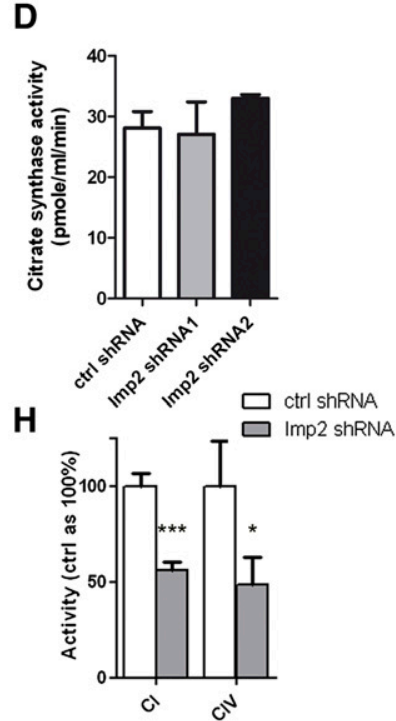

Figure 5. Imp2 depletion affects mitochondrial respiration in gliomasphere cells. (A) MitoTracker Red staining of mitochondria decreases in cells depleted of Imp2, as analyzed by FACS. DAPI-stained dead cells were excluded from the analysis. Bars represent mean values for three independent cell batches. $\left(^{\star \star}\right) P=0.0071$ and 0.0081 (paired two-tailed $t$-test), respectively. Error bars represent the SD. $(B)$ OCR is decreased by Imp2 depletion. OCR values were normalized to viable cell counts as assessed by post-measurement FACS analysis of Calcein AM staining. Results for three cell batches are shown. Imp2 shRNA1: $\left(^{\star \star}\right) P=0.005,\left({ }^{\star}\right) P=0.005,\left(^{\star \star}\right) P=0.0038$; Imp2 shRNA2: $\left.\left(^{\star}\right) P=0.0114,\left.\right|^{\star \star}\right) P=0.0021,\left(^{\star \star \star}\right) P=0.0004$ (unpaired two-tailed $t$-test). $(C)$ The OCR is higher in freshly isolated $\mathrm{CD}_{133^{+}}$than in CD133- cells. Results for two freshly obtained surgical biopsies are shown. $\left(^{\star}\right) P=0.0355 ;\left(^{\star \star \star \star}\right) P<0.0001$ (unpaired two-tailed $t$-test). (D) Citrate synthase activity upon Imp2 knockdown. Representative values of two experiments with three different cell batches are shown. $P>0.05$, not significant. $(E)$ ATP content upon Imp2 knockdown. Representative values of two experiments with two different cell batches are shown. $\left(^{\star \star}\right) P=0.0024$ (two-tailed $t$-test). ( $F$ ) Respiratory CI and CIV activity is lowered by Imp2 knockdown, as shown by BN-PAGE and in-gel activity assay. Forty micrograms of the mitochondrial extracts was loaded onto the gel. Representative results of three independent experiments with three different cell batches are shown. Coomassie staining shows band intensity before the activity assay. Error bars represent the SD. $(G)$ Quantification of CI and CIV Coomassie-stained bands. No significant difference was observed. $(H)$ Quantification of CI and CIV in-gel activity. $\left.\left(^{\star \star \star}\right) P=0.0008 ;{ }^{\star}\right) P=0.0321$ (two-tailed $t$-test). 
a Blue Native PAGE (BN-PAGE) that allows in-gel measurements of respiratory complex activity (Nijtmans et al. 2002). Mitochondrial extracts from GBM spheres stably infected with Imp2 shRNA showed a marked decrease in both CI and CIV activity (Fig. 5F-H).

Mitochondrial respiration generates reactive oxygen species (ROS), raising the question as to whether the effect of Imp2 depletion in sphere-forming cells could be at least partially explained by ROS-induced cell death. Superoxide anion production measured by MitoSOX staining was decreased in Imp2-depleted spherogenic cells (Supplemental Fig. 4A), as was the level of 4-hydroxynonenal protein adducts, stable lipid peroxidation products that reflect chronic oxidative stress (Supplemental Fig. 4B). These observations suggest that Imp2 depletion-triggered cell death is not due to increased ROS production.

\section{OXPHOS inhibition by rotenone mimics the effects of Imp2 depletion and is crucial for energy metabolism in spherogenic GBM cells}

Our observations provide evidence that GBM spheres require an intact mitochondrial respiratory chain to survive and sustain their tumorigenic potential. To address the relative importance of OXPHOS and anaerobic glycolysis in GBM spheres, we used inhibitors of each pathway, including rotenone, which blocks CI activity, and oxamic acid, which abrogates lactate dehydrogenase. Rotenone treatment recapitulated the effect of Imp2 shRNA with disruption of sphere morphology (Fig. 6A,B) and abrogation of clonogenicity (Fig. 6C). In contrast, oxamic acid mildly increased the number of spheres in culture, as shown by post-treatment clonogenic assays (Fig. 6A-C). Inhibition of CI by addition of rotenone to the cell culture medium during measurement decreased the OCR of control shRNA-expressing cells to the level measured in Imp2-deficient cells (Fig. 6D), supporting the notion that the observed difference in OCR in Imp2depleted cells was due to a defect in OXPHOS.

Our observations thus far suggest that despite being resistant to hypoxia (Li et al. 2009), gliomaspheres heavily rely on OXPHOS as their main energy-producing pathway. To determine whether spherogenic and adherent cells possess different energy requirements, the effects of OXPHOS and anaerobic glycolysis inhibitors were assessed on primary spherogenic and adherent GBM cells obtained by culturing sphere-derived cells in serum-containing medium to induce differentiation (Singh et al. 2004). ATP levels measured after short-term treatment with rotenone were decreased only in gliomaspheres, whereas adherent cell ATP content was diminished only by oxamic acid (Fig. 6E). Proliferation assays upon addition of oxamic acid to the culture medium showed that inhibition of anaerobic glycolysis affects the growth of serum-treated GBM cells but not sphere-forming cells (Fig. 6F). In contrast, rotenone decreased proliferation of both cell types. Inhibition of respiratory CI by rotenone may not only decrease ATP levels in cells dependent on OXPHOS, but may also generate ROS, leading to induction of cell death. We therefore tested superoxide production by MitoSOX staining and ROS-dependent protein oxidation using HNE adduct assays on spheres and adherent cells treated with rotenone (Supplemental Fig. 4C,D). Similar to Imp2 depletion (Supplemental Fig. 4A), reduction of oxidative stress was observed in spherogenic cells exposed to rotenone. In contrast, rotenone increased superoxide production and protein oxidation in adherent cells, which may reflect enhanced ROS production and explain their decreased proliferation in response to rotenone.

ATP-independent inhibition of adherent cell proliferation after rotenone treatment was confirmed by oligomycin treatment. Oligomycin, which inhibits the ATPase of respiratory complex $\mathrm{V}$, had no effect on adherent cell proliferation, whereas it strongly inhibited sphere proliferation (Supplemental Fig. 4E). This observation supports the notion that spherogenic and adherent GBM cells that may represent the tumor bulk have distinct energy requirements.

The metabolic differences between adherent cells and spheres were also reflected in Imp2 knockdown phenotypes (Supplemental Fig. 5A-D). Depletion of Imp2 in adherent cultures did not affect cellular proliferation or ATP levels, although, similar to spheres, the OCR was decreased (Supplemental Fig. 4D). To verify that the OCR was not affected by merely culturing cells as spheres or adherent monolayers, we assessed the OCR of serumtreated adherent cells following trypsinization. The OCR of adherent GBM cells was not observed to change whether the measurement was made on cells that were allowed to attach to the culture plate or on cells that had been freshly resuspended (data not shown). Moreover, analysis of the mRNA repertoire bound by Imp2 in adherent cells revealed that it was almost identical to that bound to Imp2 in spheres (Supplemental Table 2). It is noteworthy that the intracellular localization of the Imp2 protein is the same in spheres and adherent cells (Supplemental Fig. 5E).

To further address the relationship between Imp2 and mitochondrial respiration, the effect of Imp2 overexpression in the immortalized astrocytic cell line SVGp12 was assessed. Introduction of exogenous Imp2 into SVGp12 cells resulted in increased Imp2-interacting protein expression; enhanced mitochondrial activity, as assessed by MitoTracker Red staining; and augmented OCR (Supplemental Fig. 6). In addition, a rescue experiment on spherogenic cells was performed. Sphere-derived cells overexpressing the coding sequence of Imp2 were transiently transfected with siRNA targeted at the $3^{\prime}$ untranslated region (UTR) of Imp2 mRNA (Supplemental Fig. 6D). OCR measurement in these cells revealed that the oxygen consumption decrease caused by Imp2 depletion can be rescued by exogenous reintroduction of the protein (Supplemental Fig. 6E). Together, these results support direct involvement of Imp2 in OXPHOS control.

The effect of hypoxia on Imp2 expression and mRNA binding and the clonogenic potential of gliomasphere-derived cells

Thus far, our results indicate that gliomasphere maintenance requires OXPHOS. However, CSCs have been identified in both hypoxic regions and vascular niches 
A
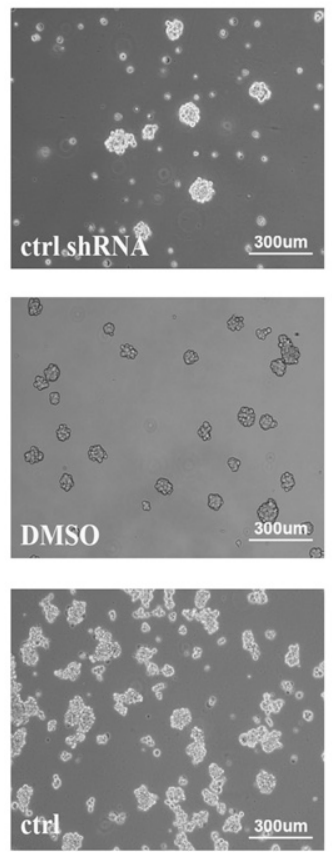

E

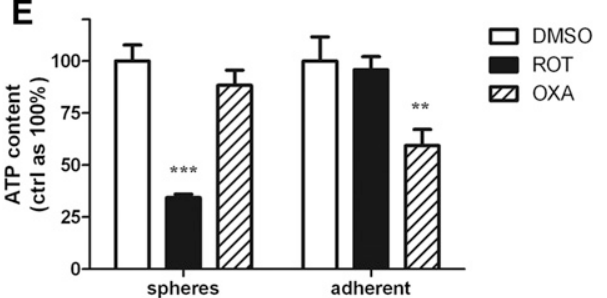

B
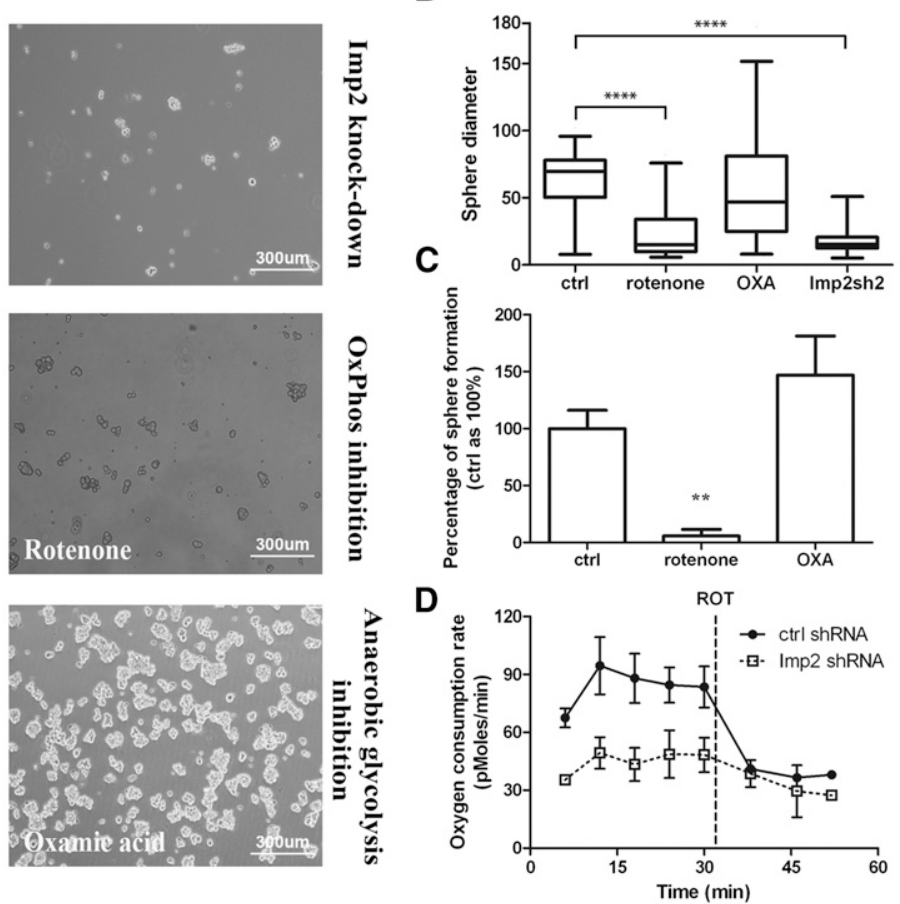

$\mathbf{F}$

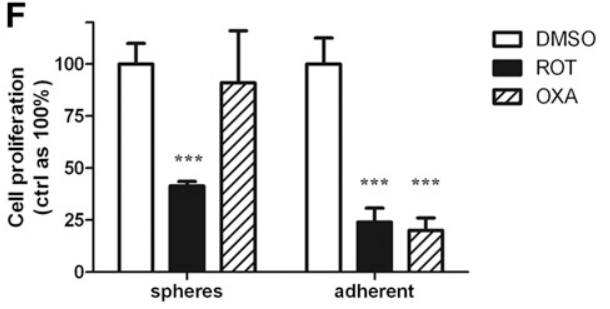

Figure 6. Inhibition of OXPHOS impairs clonogenicity and survival of gliomasphere cells. $(A)$ Inhibition of OXPHOS with $1 \mu M$ rotenone affects gliomasphere morphology in the same way as Imp2 silencing. $(B)$ Morphological changes in response to rotenone (ROT), oxamic acid (OXA), and Imp2 knockdown shown as sphere diameter measurements. $\left.{ }^{\star \star \star \star}\right) P<0.0001(n=50)$, unpaired twotailed $t$-test. $(C)$ Clonogenic potential of gliomasphere cells is decreased by rotenone treatment. $\left({ }^{\star \star}\right) P=0.0024$, unpaired two-tailed $t$-test. In contrast, anaerobic glycolysis inhibition by $25 \mathrm{mM}$ oxamic acid does not significantly affect the clonogenic potential of GBM CSCs. Representative results for one out of three cell batches are shown. (D) Addition of $1 \mu \mathrm{M}$ rotenone (indicated by the vertical line) decreases the OCR of control cells to a level comparable with that measured in Imp2-depleted cells. (E) Rotenone decreases ATP content in spheres but not in adherent cells, whereas oxamic acid affects adherent cells but not spheres. ${ }^{\star \star \star} \mid P=$ $\left.0.0001 ;\left.\right|^{\star \star}\right) P=0.0074$, unpaired two-tailed $t$-test. Representative results for one out of two cell batches are shown. $(F)$ Oxamic acid affects proliferation of only adherent cells as measured in a BrdU incorporation assay; rotenone inhibits proliferation of both gliomasphere and adherent cells. $\left.{ }^{\star \star \star}\right) P=0.0006$ (DMSO vs. rotenone in spheres), 0.0008 (DMSO vs. rotenone in adherent cells), and 0.0001 (DMSO vs. oxamic acid in adherent cells), unpaired two-tailed $t$-test. Representative results for one out of two cell batches are shown. Error bars represent the SD.

in primary GBM (Li et al. 2009; Anido et al. 2010; Charles et al. 2010). We therefore investigated the effect of hypoxia on Imp2 expression and function. Imp2 protein expression was assessed in both normoxic and hypoxic as well as high- and low-glucose culture conditions. None of these conditions induced a significant variation in Imp2 expression (Supplemental Fig. 7A). Based on our observations that impairment of OXPHOS by Imp2 depletion or rotenone treatment in normoxic conditions inhibits gliomasphere formation, we assessed the effect of both measures on gliomasphere formation under hypoxic conditions. Neither the effect of Imp2 depletion nor that of rotenone on sphere-forming capacity was altered by hypoxia (Supplemental Fig. 7B), suggesting that $1 \%$ oxygen is sufficient for gliomaspheres to maintain OXPHOS. As expression of Imp2 in hypoxic conditions remained unchanged, we assessed possible hypoxia-induced alterations in its mRNA-binding ability. qRT-PCR analysis of Imp2 RIP fractions revealed that several of the identified Imp2 target mRNAs were more highly enriched when gliomaspheres were cultured under hypoxic than under normoxic conditions (Supplemental Fig. 7C), suggesting a higher efficacy of mRNA binding by Imp2 in hypoxia. 
Janiszewska et al.

Imp2 regulates OXPHOS by $m R N A$ delivery to mitochondrial polysomes and by control of respiratory CI assembly

The mechanism by which Imp2 controls mitochondrial energy production could involve stabilization of its target mRNAs and/or their subcellular localization to the vicinity of mitochondria, in addition to the assembly and function of CI. To determine whether degradation of its target mRNAs depends on Imp2 expression, cells overexpressing or depleted of Imp2 were treated with actinomycin D to block mRNA polymerization. Degradation of selected target transcripts was then assessed at several time points for a total of $12 \mathrm{~h}$ (Fig. 7A). Although the expression level of the target RNAs was dependent on that of Imp2, the slope of the decay curve was not significantly altered, suggesting that Imp2 does not play a major role in its target mRNA stability. Next, Imp2 localization was assessed by Western blot analysis of subcellular GBM sphere-forming cell fractions using an anti-Imp2 antibody. Imp2 was found in the cytoplasm as well as in intact mitochondria, where NDUFS3 is also located (Fig. 7B, lane 2). Proteinase K treatment of mitochondria abolished Imp2 detection, consistent with its localization on the mitochondrial surface (Fig. 7B, lane 3). The partial degradation of NDUFS3 observed following proteinase $\mathrm{K}$ treatment suggests that a fraction of its cellular content is localized on the mitochondrial surface, where interaction with Imp2 may occur.

NDUFS3 and NDUFS7 both interact with Imp2 and are found in a subcomplex that initiates CI assembly (Vogel et al. 2007), suggesting that Imp2 may be implicated in early CI assembly events. BN-PAGE followed by Western blot analysis of NDUFS3 in control and Imp2-depleted cells revealed a decrease in the amount of high-molecularweight $\mathrm{CI}$ in the latter, together with the appearance of more prominent lower-molecular-weight bands that correspond to CI subcomplexes (Fig. 7C, left panel). To verify that the high-molecular-weight CI depletion was not merely a reflection of a decrease in NDUFS3 expression, we assessed the expression of the CI subunit ND2 that does not interact with Imp2. A similar effect was
A

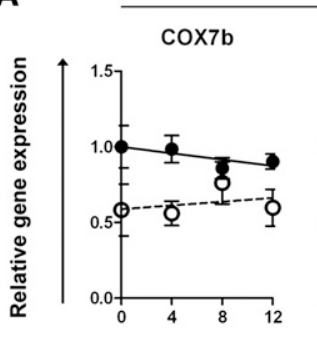

$\rightarrow$ ctrl $\rightarrow$ Imp2 shRNA

B

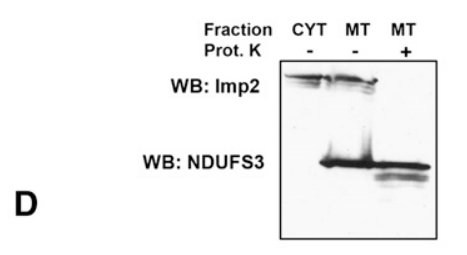

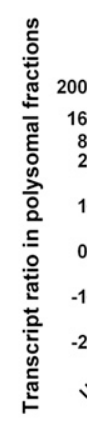

-r. pMSCV_Imp2

Imp2 knock-down

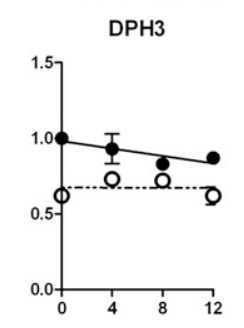

Actinomycin D treatment (h)

\section{C}
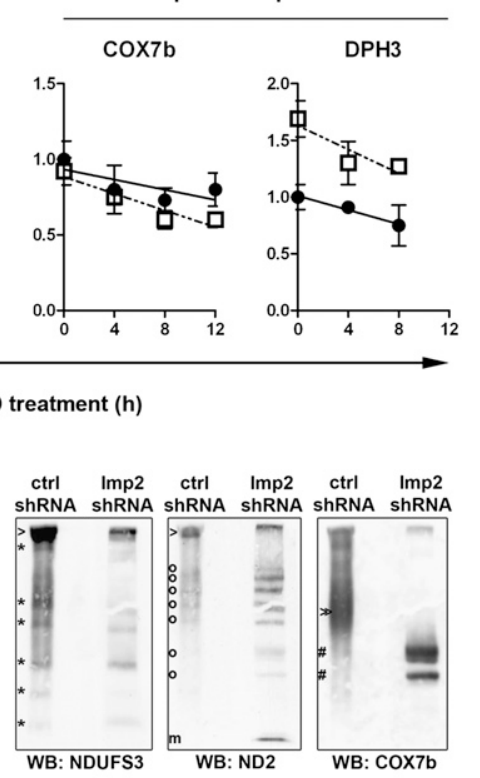

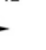

transcripts are shown. (B) Imp2 localizes to the surface of mitochondria as illustrated by subcellular fractionation. (CYT) Cytosolic fraction; (MT) isolated mitochondria. Western blot analysis of Imp2 and NDUFS3 shows the disappearance of the Imp2 band following proteinase $\mathrm{K}$ treatment of intact mitochondria. $(C)$ Assembly of respiratory CI and CIV is impaired by Imp2 knockdown; BN-PAGE followed by Western blot (WB) using anti-NDUFS3, anti-ND2, and anti-COX7b antibodies. Imp2 knockdown depleted the high-molecular-weight CI (NDUFS3 WB) and increased accumulation of its intermediate species (ND2 WB). CIV assembly (COX7b WB) was also altered. (>) CI holocomplex; ${ }^{*}$ ) CI subcomplexes containing NDUFS3 (pattern corresponding to that published by Vogel 2007); (o) CI subcomplexes containing ND2; $(\mathrm{m})$ monomeric ND2; (>>) CIV holocomplex; (\#) CIV subcomplexes. Holocomplex band localization was verified with Native Mark marker (Invitrogen) and the in-gel activity assays. $(D)$ Imp2 delivers mRNA to mitochondria-bound polysomes; qRT-PCR was performed on a mitochondria-bound polysomal fraction and cytosolic polysomes isolated from SVGp12 astrocytes overexpressing Imp2 or infected with empty vector. Ratios of transcript content in cytosolic to that in mitochondria-bound polysomes are presented. The ratio value for UCP2, a mitochondria-bound polysome-associated transcript, was set as the 0 value. Values below 0 indicate higher mitochondrial than free polysome association of a given transcript. Controls were 12S (mitochondrial rRNA for mitochondria purification efficiency), UCP2 (a mitochondrial protein that is not a direct target of Imp2), and nestin (a cytosolic protein that is not a direct target of Imp2). Representative values of two independent Imp2 overexpression experiments are shown. 
observed in Imp2-depleted cells, as illustrated by a depletion of high-molecular-weight $\mathrm{CI}$ and the appearance of numerous lower-molecular-weight bands that correspond to CI subcomplexes (Fig. 7C, middle panel). These observations suggest that $\mathrm{CI}$ assembly requires Imp2.

Although much less is known about CIV than about CI assembly, we observed that COX $7 \mathrm{~b}$ displays markedly different migration, as assessed by Western blot analysis of lysates from control and Imp2-depleted cells, consistent with a possible defect in CIV assembly in the latter (Fig. 7C, right panel).

To determine whether Imp2 may serve as a delivery system for nuclear-encoded transcripts to mitochondrial polysomes, subcellular fractionation of extracts from astrocytes overexpressing Imp2 was performed. Using qRT-PCR, ratios between transcripts in the cytosolic and mitochondria-bound polysomal fractions in control and Imp2-overexpressing cells were calculated. High Imp2 expression was associated with increased amounts of Imp2-bound transcripts on mitochondria-bound polysomes (Fig. 7D). These results indicate that Imp2 facilitates localization of nuclear-encoded mRNA to the vicinity of mitochondria, where subsequent translation and insertion into mitochondrial membranes may occur.

\section{Discussion}

Our study provides the novel observations that an mRNAbinding oncofetal protein, Imp2, controls OXPHOS in GBM cells by binding both respiratory CIV transcripts and CI proteins. Imp2 may thereby play an essential role in the survival of cells that use OXPHOS as their primary energy source.

Imp2 remains the least-studied member of its family. In human tissues, low levels of Imp2 transcripts have been detected in adult bone marrow, colon, kidney, salivary glands, small intestine, and gonads (Hammer et al. 2005), whereas its protein has thus far been reported only in the islets of Langerhans (Dai et al. 2011) and gonads (Hammer et al. 2005). In mice, Imp2 is found in a broad range of adult tissues, its highest expression being observed in the brain (Dai et al. 2011). Our study shows that Imp2 is absent in normal adult human brains and primary astrocytes (data not shown). However, it was detected in the SVZs of fetal brains where stem cells reside.

Among GBM molecular subtypes, as defined by the TCGA database, Imp2 is least expressed in neural GBM, characterized by a gene expression profile that most closely resembles normal brain tissue. In contrast, high Imp2 expression bears a prognostic value for patients with the proneural GBM subtype whose gene expression profile resembles that of tumors associated with elevated CD133 expression (Joo et al. 2008). Moreover, the gene expression profile of proneural GBM shares similarities with that of fetal neural stem cells, suggesting that it may contain an elevated proportion of stem-like cells (Lottaz et al. 2010). These results are consistent with our in vitro studies, showing that Imp2 is not exclusively expressed in CSC-enriched GBM subpopulations, but that it may play a crucial role in their maintenance.
In the present study, we used gliomasphere cultures as a model for highly tumorigenic subpopulations that display self-renewal capability and showed that Imp2dependent OXPHOS is required for the maintenance of these cells. Sphere-derived CD133 ${ }^{+}$cells displayed higher oxygen consumption than their $\mathrm{CD} 133^{-}$counterparts, and we further demonstrated that $\mathrm{CD} 133^{+}$cell subpopulations from fresh GBM, unexposed to in vitro culture, not only express elevated levels of Imp2, but also consume more oxygen than $\mathrm{CD} 133^{-}$cells. Although we could not perform functional assays on ex vivo $\mathrm{CD} 133^{+}$ cells without expansion in vitro and could not measure oxygen consumption of single cells that may have allowed definitive distinction between CSC and non-CSC populations, the sum of our observations supports the notion that Imp2 plays a key role in the survival and function of GBM cells that display CSC features.

The function of Imp2 as an mRNA-binding protein was first established with the demonstration that it binds IGF2 mRNA (Nielsen et al. 1999). Only one report so far has indicated the existence of other targets by showing that Imp2 can regulate cytoskeletal reorganization, which underlies the motility of human fetal myoblasts (Boudoukha et al. 2010). Interestingly, Imp2-bound transcripts in myoblasts also contain a significant proportion of species associated with mitochondrial respiration, suggesting that Imp2 may be implicated in cellular energy production independent of cell type. Another potential clue that may link Imp2 to mitochondrial functions is the observation that a single-nucleotide polymorphism within the IMP2 gene is strongly associated with type 2 diabetes (Saxena et al. 2007; Scott et al. 2007), where significant mitochondrial dysfunction is known to occur. A mechanistic link to metabolism was, in fact, established by the demonstration that Imp2 is a target of mTOR (Dai et al. 2011), a kinase complex that is tightly coupled to cellular bioenergetics (for review, see Zoncu et al. 2011). mTOR was shown to phosphorylate Imp2, which results in its increased binding to IGF2 mRNA. Defects in mTOR regulation have been associated with both cancer and type 2 diabetes, suggesting that part of the effect of aberrant mTOR pathway activation could potentially be due to altered Imp2 activity.

Of the multitude of proteins that compose the respiratory electron transport chain, only a fraction is encoded by mitochondrial DNA. The majority are encoded by nuclear DNA, and their transcripts are presumed to be transported by RNA-binding proteins to the vicinity of mitochondria, where they can be translated on mitochondria-bound polysomes and inserted into mitochondrial membranes (Suissa and Schatz 1982). Our observations indicate that Imp2 provides at least one vehicle for mRNA transport to mitochondria. Not only are numerous CIV protein-encoding transcripts bound to Imp2, but their quantity is augmented in polysomes associated with mitochondria upon Imp2 overexpression in an astrocytic cell line. The relevance of Imp2-mediated delivery of these transcripts is supported by the finding that depletion of Imp2 leads to impaired CIV assembly and loss of CIV activity. Thus, one hitherto unknown function of 
Imp2 is the regulation of respiratory CIV assembly and function by delivering at least some of its building blocks to mitochondrial polysomes.

Involvement in mitochondrial functions is further supported by the observation that Imp2 binds several proteins of respiratory CI. Unexpectedly, CI subunitbound Imp2 was detected on the mitochondrial surface, suggesting that Imp2 binds CI proteins prior to their translocation into mitochondria. The interaction may transiently anchor Imp2 to the mitochondria, possibly facilitating the delivery and local translation of Imp2bound transcripts. Repression of Imp2 resulted in depletion of high-molecular-weight CI bands and impaired CI function, as assessed by in-gel activity assays. Thus, the interaction of CI proteins with Imp2 appears to be required for $\mathrm{CI}$ assembly and activity. The absence of Imp2-bound CI transcripts suggests that Imp2 regulates CI function by physically interacting with its subunit components. CI is composed of several subcomplexes, and its assembly in mammalian cells is a multistep process that remains incompletely understood (Vogel et al. 2007). Interestingly, NDUFS3 and NDUFS7, the subunits of CI bound by Imp2, form the earliest and smallest subcomplexes, suggesting that Imp2 could be implicated in the initiation of CI assembly (Vogel et al. 2007). Numerous chaperones are believed to be required for CI assembly and translocation of its components from the cytoplasm to the inner mitochondrial membrane, but only a few have been identified thus far (for review, see Rehling et al. 2004). Our present observations are consistent with the possibility that Imp2 may be a CI subunit chaperone that orchestrates CI assembly and/or translocation into mitochondria while at the same time delivering CIV transcripts. By controlling both CI and CIV assembly and function, Imp2 may play a prominent role in the regulation of cellular energy production, and its expression may therefore be crucial to cells that derive their energy primarily from OXPHOS.

Gliomasphere dependence on OXPHOS predicts that GBM CSCs should be located in highly oxygenated regions of the tumor. Although they indeed primarily reside in perivascular areas (Calabrese et al. 2007; Currle and Gilbertson 2008), GBM CSCs are also found in hypoxic tumor zones (Li et al. 2009). Accordingly, Imp2 expression was elevated in GBM cells around blood vessels and in pseudopalisading cells surrounding necrosis. GBM pseudopalisades are believed to be composed of cells that migrate away from ineffective or obstructed vasculature (Rong et al. 2006). It is therefore plausible that the disruption of blood supply following thrombosis caused CSCs to leave their primary perivascular niche and migrate to constitute new niches characterized by the pseudopalisading phenotype. Consistent with this notion and the observation that GBM CSCs are resistant to hypoxia (Ezhilarasan et al. 2007; Li et al. 2009), we found that Imp2 levels remain constant under normoxic and hypoxic culture conditions and that hypoxia does not affect GBM clonogenic potential (Supplemental Fig. $7 \mathrm{~A}, \mathrm{~B})$. Interestingly, target mRNA binding by Imp2 is enhanced in hypoxic conditions (Supplemental Fig. 7C), suggesting a possible mechanism for maintaining OXPHOS in a low-oxygen environment. Active use of OXPHOS by cancer cells should not be inhibited by hypoxic conditions, as oxygen concentration in a hypoxic microenvironment has been determined to be in the range of 8-57 $\mu \mathrm{M}$ (Vaupel et al. 1989; Görlach and Acker 1994; Sutherland 1998), which is 10-fold higher than the limiting concentration for the $\mathrm{O}_{2}$ dissociation constant for cytochrome c oxidase (Mason et al. 2006). The dependence of GBM CSC-enriched subpopulations on OXPHOS suggested by our results may thus be independent of their location within the tumor.

Bioenergetic reprogramming constitutes part of the profound alterations induced by transformation. The ensuing changes in growth patterns and augmented anabolism impose increased energy requirements on transformed cells compared with their normal counterparts (Hanahan and Weinberg 2011). The longstanding view, first proposed by Warburg (Vander Heiden et al. 2009), is that these requirements are primarily fulfilled by glycolysis even in the presence of an adequate oxygen supply. Glycolysis provides rapid but inefficient energy production, generating 2-4 mol of ATP per mole of glucose. However, glucose, along with glutamine, is the major source of carbon, free energy, and reducing equivalents required to support cell growth and division. Thus, a substantial portion of glucose is likely to be used to generate macromolecular precursors of fatty acid, nonessential amino acid, and nucleotide synthesis in proliferating cells. Nevertheless, glycolysis is not a major energy source in all cancer cells (Jose et al. 2011), and those that have not sustained major mitochondrial damage or corresponding DNA mutations may alternate between OXPHOS and glycolysis, depending on their state and microenvironment. In our hands, spherogenic GBM cells proliferated more slowly than their adherent progeny. Such slowly proliferating cells may use the more efficient OXPHOS pathway that yields $36 \mathrm{~mol}$ of ATP per mole of glucose. Oxygen supply, required for OXPHOS, may be more readily available for GBM CSCs than for more differentiated GBM cells, based on the observations that GBM CSCs reside in the proximity of blood vessels (Calabrese et al. 2007; Currle and Gilbertson 2008) and that they may even generate tumor endothelium (Ricci-Vitiani et al. 2010; Wang et al. 2010). As CSC progeny proliferate, increased anabolic requirements and/or genetic mutations that inactivate mitochondria could force the cells to switch to a predominantly glycolytic metabolism. It is therefore plausible that hierarchical cellular organization within a tumor is also reflected in metabolic differences between cancer cell subpopulations.

Imp2 expression is not restricted to spherogenic tumorinitiating and self-renewing cells, but is also present in adherent cultures that may represent the tumor bulk but have lost tumorigenic potential. Importantly, the mRNAbinding repertoire of Imp2 was shared among normal neural progenitors, gliomaspheres, and adherent GBM cells. Moreover, its overexpression in normal astrocytes increased their oxygen consumption, and, similar to spherogenic cells, adherent GBM cells depleted of Imp2 
displayed decreased OCR. The function of Imp2 therefore appears to be neither cell type- nor transformation-dependent. However, whereas an OCR decrease in gliomaspheres was accompanied by a chronic $20 \%$ drop in intracellular ATP that significantly affected survival, ATP levels in adherent cells remained unaltered in the face of decreased OCR. These cells therefore appeared to be resistant to OXPHOS inhibition and to have shifted their energy dependence toward glycolysis, consistent with the Warburg effect. The dichotomy between spherogenic and adherent cells regarding their dependence on OXPHOS or glycolysis was not related to the high oxygen and glucose levels of in vitro culture, as it remained unaltered when the cells were subjected to hypoxic, low-glucose conditions that more closely reflect the primary tumor microenvironment. Metabolic differences between primitive GBM CSCs and more differentiated cells have been reported by others (Wakimoto et al. 2009; Vlashi et al. 2011), supporting our observations that cells with CSC features depend on OXPHOS. Importantly, these differences were also observed ex vivo on CSC subpopulations freshly dissociated from surgically removed tumor specimens. Whether dependence on OXPHOS can be generalized to tumorigenic cell populations with CSC features, irrespective of tumor type, remains to be explored.

Taken together, our observations provide new insight into the function of Imp2 as a respiratory chain CIV mRNA-binding protein and a putative CI chaperone that regulates OXPHOS and appears to be critical for GBM sphere-forming cell survival and function. These findings may have important clinical implications, as the selective expression of Imp2 in GBM may render it a potentially attractive novel therapeutic target for depletion of tumorigenic cells with CSC features.

\section{Materials and methods}

Tumor samples, gliomaspheres, adherent cells, human neural progenitor cell culture, clonogenic assays, and sphere diameter measurement

Experimental procedures were performed as previously described (Suvà et al. 2009). Briefly, surgical biopsies from three GBM patients (in accordance with HUG protocol 04-113) were dissociated to single-cell suspension and cultured in DMEM/F12 (Gibco), 20\% BIT (Stem Cell Technologies), $10 \mathrm{ng} / \mathrm{mL}$ recombinant human epidermal growth factor (EGF) (Invitrogen), $10 \mathrm{ng} /$ $\mathrm{mL}$ recombinant human basic fibroblast growth factor (FGF) (Invitrogen), and 1\% penicillin/streptomycin (Gibco). As previously described (Galli et al. 2004; Singh et al. 2004; Lee et al. 2006; Beier et al. 2007), nonadherent cellular spheroids obtained in these growth conditions were considered as cultures enriched for CSCs and were used for subsequent experiments. Gliomaspheres were retained in culture for no longer than 15 passages, and it has been reported that CSCs can be sustained in spherogenic cultures for up to 25 passages (Clement et al. 2010). Adherent cell cultures were obtained by spheroid dissociation and culture in serum-containing DMEM-F12 (Gibco) supplemented with $10 \%$ FBS (Gibco) and 1\% penicillin/streptomycin (Gibco). The human astrocyte cell line SVGp12 was cultured in RPMI (Gibco) supplemented with 10\% FBS (Gibco) and 1\% penicillin/streptomycin (Gibco). The human neural progenitor cells hNP1 (Aruna Biomedical) were cultured according to the provider's protocol on Matrigel-coated plates in AB2 basal neural medium supplemented with ANS neural medium supplement (Aruna Biomedical), 50 mg/mL FGF (Invitrogen), $200 \mathrm{mM}$ glutamine (Gibco), $10 \mu \mathrm{g} / \mathrm{mL}$ LIF (Milipore), and $1 \%$ penicillin/ streptomycin (Gibco).

For clonogenic assays, disaggregated spheroids were plated as single cells at a density of one cell per well in 96-well plates, three plates per condition, and cultured for $2 \mathrm{wk}$ in serum-free medium.

Sphere diameter measurements were done with ImageJ software. For each condition, images of 50-100 spheres were analyzed.

\section{Survival analysis}

Imp2 expression in GBM samples stratified according to TCGA criteria was obtained from the combined data set of Verhaak et al. (2010). Samples were divided into "high" (top 50\%) and "low" (bottom 50\%) groups based on Imp2 expression. For Kaplan-Meier survival analysis, only patients that had received both chemotherapy and radiation therapy were considered, and proneural samples were selected based on assignment from Verhaak et al. (2010). All calculations were performed with the $\mathrm{R}$ statistical computation package.

\section{CD133 cell isolation}

Surgical biopsies from six GBM patients were immediately dissociated to single-cell suspensions with Brain Tumor Dissociation Kit P (Miltenyi), according to the manufacturer's protocol. Dead cell removal was performed on MACS columns (Miltenyi), and subsequently, cell sorting was performed using a CD133 Cell Isolation kit (Miltenyi).

\section{Chemical compounds and treatments}

Inhibition of OXPHOS was achieved by $1 \mu \mathrm{M}$ rotenone (Sigma). Inhibition of complex V ATPase was performed with $5 \mu \mathrm{g} / \mathrm{mL}$ oligomycin. The lactate dehydrogenase inhibitor oxamic acid was used at $25 \mathrm{mM}$ to block glycolysis. Treatments were applied for $72 \mathrm{~h}$. For ATP content measurement, treatment time was shortened to $0.5 \mathrm{~h}$.

For mRNA degradation studies, $10 \mu \mathrm{g} / \mathrm{mL}$ actinomycin $\mathrm{D}$ (Sigma) was added to cell growth medium. RNA was collected after $0,4,8$, and $12 \mathrm{~h}$ of treatment.

Imp2 knockdown and retroviral infection

shRNA sequences targeting Imp2 were sh1 sense strand (5'-GA TCCACCAAACTAGCCGAAGAGATTCAAGAGATCTCTTC GGCTAGTTTGGTTTTTTTACGCGTG-3') and antisense strand (5'-AATTCACGCGTAAAAAAACCAAACTAGCCGAAGAGA TCTCTTGAATCTCTTCGGCTAGTTTGGTG-3') and sh2 sense strand (5'-GATCCGCGGAAAGAACCATCACTGTTTCAAGA GAACAGTGATGGTTCTTTCCGTTTTTTACGCGTG3') and antisense strand (5' -AATTCACGCGTAAAAAACGGAAAGAA CCATCACTGTTCTCTTGAAACAGTGATGGTTCTTTCCG CG-3').

Sense and antisense oligonucleotides were annealed to form duplexes and inserted into the pSIREN-Retro Q retroviral vector (BD Biosciences Clontech) according to the manufacturer's recommendations. Imp2 or control shRNA plasmids were transfected into GP2 packaging cells to produce the virus used to infect target gliomaspheres. Viral supernatant was concentrated by ultracentrifugation using a SW28 rotor (Beckman Coulter) at $19,500 \mathrm{rpm}$ for $90 \mathrm{~min}$. Concentrated virus was added to 
dissociated spheres. Forty-eight hours later, cells were selected for puromycin $(2 \mu \mathrm{g} / \mathrm{mL})$ resistance for $5 \mathrm{~d}$. The efficiency of Imp2 depletion was verified by qRT-PCR and Western blot analysis. All experiments with Imp2-depleted cells were performed immediately after the end of the selection period.

\section{Imp2 overexpression}

The PCR product generated with the primers Fwd (5'-GAAG ATCTTCCCACCATGATGAACAAGCTTTACATC-3') and Rev (5'-CGGAATTCTCACTTGCTGCGCTGTGAGGCGACT-3') on GBM CSC genomic DNA was cloned into the pMSCV_puro vector (Clontech). The human immortalized astrocytic cell line SVGp12 was infected with pMSCV_Imp2 or empty vector containing virus according to standard protocols. Cells were selected for puromycin $(2 \mu \mathrm{g} / \mathrm{mL})$ resistance for $7 \mathrm{~d}$.

\section{Rescue experiment}

Spherogenic cells were infected with pMSCV_Imp2- or empty vector-containing virus, selected for puromycin $(2 \mu \mathrm{g} / \mathrm{mL})$ resistance for $5 \mathrm{~d}$, dissociated, and transfected with Imp2 siRNA (5'-CACCTGACAGAATGAGACCTT-3') specific for the 3' UTR mRNA region or ctrl siRNA. Seventy-two hours post-transfection, the Imp2 protein expression level was determined, and cells were subjected to OCR measurement.

\section{NOD/SCID mouse xenotransplantation and survival analysis}

The in vivo experiments were conducted as described previously (Suvà et al. 2009). Intracranial injections of 500 cells (unless indicated otherwise) from gliomaspheres at coordinates $x=-2$, $y=0$, and $z=-2$ relative to bregma point were performed with a stereotactic apparatus (Kopf Instruments). The procedure was approved by the Etat de Genève, Service Vétérinaire, authorization number 1007/3337/2. Six NOD/SCID mice were used per condition. Survival analysis significance was calculated with a log-rank test.

\section{Immunohistochemistry}

Paraffin-embedded sections of gliomas and normal brains were stained with mouse anti-human Imp2 antibody (1:50 dilution; Abcam). Horseradish peroxidase (HRP) staining was performed using biotin-conjugated rabbit anti-mouse IgG (Vector Laboratories) and revealed with a DAKO DAB kit (DAKO).

\section{Immunofluorescence}

Gliomaspheres or adherent GBM cells were fixed with 4\% PFA, washed, permeabilized with $3 \%$ Triton X-100, incubated with anti-Imp2 antibody (1:100, $0.5 \mu \mathrm{g} / \mathrm{mL}$; Abcam) for $30 \mathrm{~min}$ followed by donkey anti-mouse Alexa488 antibody (1:1300; Molecular Probes), and mounted in 1:1000 DAPI in mounting medium (ThermoShandon). Antibody specificity was compared with the isotype-matched control antibody. Images were acquired with a Leica SP5 AOBS confocal microscope at the Imaging Core Facility of the University of Lausanne. The acquisition was performed in sequential mode to avoid dye cross-talk. Three-dimensional reconstruction of sphere staining was done with Imaris software.

Paraffin-embedded sections of GBM and embryonal brains were stained with anti-Imp2 (1:50; MBL Ribonomics), antiNestin (1:200; Milipore), anti-GFAP (1:500; Dako), or anti-SSEA-1 (1:100; Stemgent) antibody. Secondary antibodies used were donkey anti-rabbit Alexa488 (1:1300; Molecular Probes) and donkey anti-mouse Alexa594 (1:1300; Molecular Probes), respectively.

\section{FACS}

Expression of CSC-associated cell surface markers was analyzed by FACS. Single-cell suspensions were stained with singlelabeled antibody and analyzed in a FACScalibur apparatus (Becton Dickinson). Antibodies used were anti-CD133/1-phycoerythrin antibody (AC133/1, 1:10; Miltenyi), isotype control mouse $\operatorname{IgG}_{2 b}$-phycoerythrin (1:10; Miltenyi), anti-SSEA1-FITC (1:10; BD Pharmingen), and isotype control mouse IgМк-FITC (1:10; BD Pharmingen).

\section{$q R T-P C R$}

qRT-PCR was performed as described previously (Suvà et al. 2009). For normalization, the $18 \mathrm{~S}$ probe was used as the endogenous control. qRT-PCR primers are presented in Supplemental Table 1.

\section{Western blot}

Western blotting was performed according to standard procedures. The following antibodies were used: anti-Imp2 $(0.5 \mu \mathrm{g} /$ $\mathrm{mL}$; Abcam), anti-NDUFS3 (0.5 $\mu \mathrm{g} / \mathrm{mL}$; Abcam), anti-NDUFS7 $(0.5 \mu \mathrm{g} / \mathrm{mL}$; Abcam), anti-NDUF3 $(0.5 \mu \mathrm{g} / \mathrm{mL}$; Abcam), antiCox $7 \mathrm{~b}(0.5 \mu \mathrm{g} / \mathrm{mL}$; Abcam), anti-caspase 3 p20 (0.2 $\mu \mathrm{g} / \mathrm{mL}$; Santa Cruz Biotechnology), anti- $\beta$-actin $(1.65 \mu \mathrm{g} / \mathrm{mL}$; Sigma $)$, and anti$\alpha$-tubulin $(0.025 \mu \mathrm{g} / \mathrm{mL}$; Calbiochem). Secondary antibodies were HRP-conjugated goat anti-mouse (GE Healthcare), mouse anti-rabbit (DAKO), and rabbit anti-goat (DAKO) antibodies. Imp2 was detected as a doublet. However, no cross-reactivity with Imp1 or Imp3 was found (data not shown).

\section{Proliferation assay and cell cycle analysis}

Proliferation was assessed with Cell Proliferation ELISA BrdU colorimetric (Roche) according to the manufacturer's protocol. For cell cycle analysis, cells were resuspended in $10 \mathrm{ng} / \mathrm{mL}$ propidium iodide with $1 \%$ IGEPAL, vortexed, incubated overnight at $4^{\circ} \mathrm{C}$, and analyzed by FACS.

\section{Intact mitochondria isolation and proteinase $\mathrm{K}$ treatment}

Intact mitochondria were isolated from $20 \times 10^{6} \mathrm{GBM}$ CSCs with Mitochondria Isolation Kit for Cultured Cells (Pierce). To remove proteins from the mitochondrial surface, mitochondrial pellets were resuspended in buffer $\mathrm{C}$ (Mitochondria Isolation Kit for Cultured Cells, Pierce) and treated with $50 \mu \mathrm{g} / \mathrm{mL}$ proteinase $\mathrm{K}$ (Sigma) for $30 \mathrm{~min}$ on ice and spun down at 10,000 $\mathrm{g}$ for $10 \mathrm{~min}$.

\section{RIP-ChIP assay}

We used $30 \times 10^{6}$ spherogenic or adherent cells per experiment. Imp2-bound RNA immunoprecipitation was performed according to the RiboCluster Profiler RIP assay kit protocol (MBL Ribonomics). Eluted RNA was analyzed at the DNA Array Facility, Lausanne, using Affymetrics Arrays. RIP-ChIP results generated from three different primary cultures were compared. Probe sets showing a false discovery rate of $<0.05$ and logarithmic fold changes of $>2$ in all three samples were subjected to further analysis. Gene ontology annotations obtained for those probes were considered as overrepresented when the $P$-value of an exact one-tailed Fisher test was $<0.00005$.

\section{RIP assay}

Imp2-bound RIP was performed according to the RiboCluster Profiler RIP assay kit protocol (MBL Ribonomics) from $20 \times 10^{6}$ 
hNP1 cells and two spherogenic cell batches cultured in hypoxic conditions for $5 \mathrm{~d}$. Eluted RNA was analyzed by qRT-PCR.

\section{Pull-down assay}

Spherogenic cells $\left(80 \times 10^{6}\right)$ were suspended in PBS with a protease inhibitor cocktail (Roche), centrifuged, resuspended in 5 vol of RIP assay lysis buffer (without addition of DTT), and sonicated for $10 \mathrm{sec}$. The lysate was centrifuged at $3500 \mathrm{rpm}$ for 5 $\min$ at $4^{\circ} \mathrm{C}$. Agarose protein-A beads (GE Healthcare), previously washed with wash buffer from the RIP assay kit without DTT, were added to the supernatants and discarded after $2 \mathrm{~h}$ of preclearing. Incubation with $20 \mu \mathrm{g}$ of anti-Imp2 (MBL Ribonomics) or isotype-matched control antibody was performed overnight at $4^{\circ} \mathrm{C}$, followed by incubation with the beads for $4 \mathrm{~h}$. The beads were washed, and mass spectrometry analysis was performed at the Protein Analysis Facility of Faculty of Biology and Medicine, University of Lausanne.

\section{PLA}

Adherent GBM cells grown on 16-well glass chamber slides (LabTek) until confluence were transfected with a COX7b 3' UTRdesigned oligo RNA (5'-TCATCCCAGCTGGTGTAATAATGA ATTGTTTAAAAAACAGCTCATAATTGATGCCAAATTAAA GCAC-3') or VSV oligo RNA, (5' -ACATAAAAAGCTTTTTAA CCAAGCAAGAATGAAGTATCGTATCTAATTAATTCCGAT GATCAATATG-3'), each containing a $5^{\prime}$-terminal biotin tag, using DOTAP reagent (Biontex) according to the manufacturer's protocol. Six hours post-transfection, cells were fixed with $4 \%$ PFA, permeabilized with $0.3 \%$ Triton X-100 in PBS, and processed according to the Duolink In Situ PLA assay protocol (Olink Biosciences). Anti-Imp2 (1:100; Abcam) and anti-biotin (1:5000; Pierce Thermo Scientific) antibodies were used. Reaction without either anti-Imp2 or anti-biotin antibody provided controls. Samples were analyzed with a Zeiss LSM 710 Quasar confocal microscope.

\section{MitoTracker assay}

Cells were washed with PBS, resuspended in prewarmed medium containing $250 \mathrm{nM}$ MitoTracker Red CM-H2XRos (Molecular Probes) or DMSO, and incubated for $30 \mathrm{~min}$ at $37^{\circ} \mathrm{C}$. Cells were then washed and resuspended in PBS and analyzed by FACS.

\section{OCR/ECAR measurement}

Twenty-thousand to 50,000 cells per well were seeded in XF24 cell culture microplates (Seahorse Bioscience). For each cell type, five replicates were made. Measurements for spherogenic cells were made immediately after sphere disruption and viable cell counting (Trypan blue exclusion), and, following the assay, cell viability was verified (Calcein AM staining [1:200, $2 \mathrm{mg} / \mathrm{mL}$; Calbiochem] analyzed by FACS). For adherent cells, measurements were made $12-16 \mathrm{~h}$ after seeding, and uniformity in cell proliferation was verified using a Cell Proliferation ELISA BrdU colorimetric assay (Roche).

\section{ATP content measurement}

Gliomaspheres or adherent cells were disaggregated or detached, respectively, and seeded in three replicates at 50,000 viable cells per well in black-wall clear-bottom 96-well plates. Immediately after plating cells, CellTiter-Glo reagent (Promega) was added, and luminescence was measured in a SynergyMx instrument (BioTek).

\section{Citrate synthase activity measurement}

Equal numbers of viable cells were lysed with Cell Lytic M reagent (Cytrate Synthase Assay kit, Sigma), and samples were prepared according to the manufacturer's protocol. Calculations of citrate synthase activity from sample absorbance were done as described by the manufacturer.

\section{MitoSOX assay}

Superoxide production was assessed by MitoSOX Red (Molecular Probes) staining. Cells were washed, resuspended in PBS containing $5 \mu \mathrm{M}$ MitoSOX Red or DMSO, and incubated for $30 \mathrm{~min}$ at $37^{\circ} \mathrm{C}$. Cells were then washed, resuspended in PBS, and analyzed by FACS.

\section{HNE protein adduct determination}

Oxidative stress was assessed with OxiSelect HNE-His Adduct ELISA kit (Cell Biolabs) according to the manufacturer's protocol.

BN-PAGE, in-gel activity assays, and respiratory complex assembly

Mitochondrial extract preparation, Blue Native gel electrophoresis, and in-gel activity for respiratory $\mathrm{CI}$ and CIV were performed as described (Nijtmans et al. 2002). Briefly, spheres disaggregated to single cells were resuspended in PBS with protease inhibitors and lysed with an equal volume of cold digitonin solution $(8 \mathrm{mg} / \mathrm{mL}$; Calbiochem). After $10 \mathrm{~min}$ of incubation on ice and $5 \mathrm{~min}$ of centrifugation at 14,000 rpm at $4^{\circ} \mathrm{C}$, the pellet was resuspended in $1.5 \mathrm{M}$ aminocaproic acid and $50 \mathrm{mM}$ Bis-Tris- $\mathrm{HCl}(\mathrm{pH}$ 7) supplemented with $1.7 \%$ dodecylmaltoside (Invitrogen). After $5 \mathrm{~min}$ of incubation on ice and 5 min of centrifugation at $14,000 \mathrm{rpm}$ at $4^{\circ} \mathrm{C}$, the protein concentration in the supernatant was measured, and $40 \mu \mathrm{g}$ or $100 \mu \mathrm{g}$ of sample was loaded onto a native gel. For in-gel activity assays of $\mathrm{CI}$ and CIV, gels were incubated overnight with $2 \mathrm{mM}$ Tris- $\mathrm{HCl}$ (pH 7.4), $0.1 \mathrm{mg} / \mathrm{mL} \mathrm{NADH} \mathrm{(Roche),} \mathrm{and} 2.5 \mathrm{mg} / \mathrm{mL}$ nitrotetrazolium blue (Sigma), or $0.05 \%$ diaminobenzidine tetrahydrochloride (DAB), $20 \mu \mathrm{g} / \mathrm{mL}$ catalase (Sigma), $0.1 \%$ cytochrome c (Sigma), and $7.5 \%$ sucrose in PBS, respectively. Respiratory complex assembly was determined by post-BN-PAGE Western blotting. BN-PAGE gels were blotted over a PVDF membrane in $40 \mathrm{mM}$ Tris- $\mathrm{HCl}(\mathrm{pH}$ 7) with $20 \mathrm{mM}$ sodium acetate, $2 \mathrm{mM}$ EDTA, 20\% methanol, and 0.05\% SDS (from Bolt and Mahoney $1997)$ overnight at $30 \mathrm{~V}$. To remove Coomassie stain from the membrane, the blot was dried and rinsed with methanol prior to blocking. Primary antibodies used were anti-NDUFS3 $(0.5 \mu \mathrm{g} /$ $\mathrm{mL}, \mathrm{Abcam})$ and anti-ND2 $(0.5 \mu \mathrm{g} / \mathrm{mL}$; Abcam) for CI, or antiCOX7b (0.25 $\mu \mathrm{g} / \mathrm{mL}$; Abcam) for CIV.

\section{Mitochondria-bound and free polysome extraction}

Experimental procedures were performed as previously described (Sylvestre et al. 2003). SVGp12 cells stably overexpressing Imp2 or control vector were trypsinized, incubated with 250 $\mu \mathrm{g} / \mathrm{mL}$ cycloheximide for $15 \mathrm{~min}$ at $37^{\circ} \mathrm{C}$, washed, and resuspended in extraction buffer $(0.6 \mathrm{M}$ mannitol, $30 \mathrm{mM}$ Tris/ $/ \mathrm{HCl}$, at pH 7.4, 5 mM MgOAc, $100 \mathrm{mM} \mathrm{KCl} \mathrm{[7.4} \mathrm{g],} 1$ g/L BSA, 5 mM $\beta$-mercaptoethanol, $200 \mu \mathrm{g} / \mathrm{mL}$ cycloheximide, $500 \mu \mathrm{g} / \mathrm{mL}$ heparin, protease inhibitor cocktail in DEPC-water). After addition of $100 \mu \mathrm{g} / \mathrm{mL}$ digitonin and $4 \mathrm{~min}$ of incubation on ice, cells were disrupted in a glass homogenizer (cell lysis was verified under the microscope) and spun at $2500 \mathrm{rpm}$ for $7 \mathrm{~min}$. Homogenization 
and centrifugation were repeated, and the homogenates were centrifuged twice at $2500 \mathrm{rpm}$ for $7 \mathrm{~min}$. The obtained supernatant was centrifuged at $11,000 \mathrm{rpm}$ for $30 \mathrm{~min}$. The pellet containing mitochondria with bound polysomes was washed twice with the extraction buffer. The supernatant was used to obtain free cytosolic polysomes by centrifugation over a sucrose step gradient ( $2 \mathrm{M}$ and $0.5 \mathrm{M}$ sucrose in extraction buffer) at $25,000 \mathrm{rpm}$ for $17 \mathrm{~h}$. RNA was extracted from both polysomecontaining fractions (RNeasy kit, Qiagen). qRT-PCR was performed according to standard procedures. Additional primers for Nestin (Imp2-unrelated transcript; TaqMan probe), 12S (mitochondrial rRNA; control for equal mitochondrial purification efficiency; Fwd [5'-TAGCCCTAAACCTCAACAGT-3'] and Rev [5'-TGCGCTTACTTTGTAGCCTTCAT-3']), and UCP2 (control for mitochondria-bound polysomal transcripts; Fwd [5'-CCCT TGCCACTTCACTTCTG-3'] and Rev [5'-GAGAAAGGAGGG CATGAACC-3']) were used. Relative transcript amounts were normalized to $18 \mathrm{~S}$, then a ratio of transcript amount of free to mitochondria-bound polysomes was calculated for control cells and Imp2-overexpressing cells. The UCP2 ratio was set as a cutoff, as this transcript should be enriched on mitochondria-bound polysomes. Therefore, transcripts with ratios below that of UCP2 are considered to be in higher numbers on mitochondria-bound polysomes.

\section{Statistical methods}

Microarray gene expression data for nonmalignant brains and different gliomas were obtained from Sun et al. (2006) (Gene Expression Omnibus accession no. GSE4290). Data were normalized with RMA, and probes corresponding to the same gene were collapsed to the maximum value using the GenePattern software package (Reich et al. 2006). T-test $P$-values for sample comparisons were calculated using the $\mathrm{R}$ statistical software package (http://www.r-project.org).

$P$-values were calculated with GraphPad Prism 5 software using the statistical tests indicated in the figure legends.

\section{Acknowledgments}

We thank Keith Harshman (Genomic Technologies Facility, Faculty of Biology and Medicine, University of Lausanne) for performing the microarray experiments, and Manfredo Quadroni (Protein Analysis Facility, Faculty of Biology and Medicine, University of Lausanne) for the mass spectrometry analysis. We also thank Whitney Quong, Claudio De Vito, Karine Baumer, Benoît Lhermitte, and Carlo Fusco for technical assistance and discussions, and Sylviane Trepey, Jean-Christophe Stehle, and Janine Holbeck for immunohistochemistry. This work was supported by Swiss National Foundation (FNRS) grant 310030_ 130350, NCCR Molecular Oncology, a MEDIC Foundation grant to I.S., and Oncosuisse grant BIL-KFS-02590-02-2010 to M.S. P.P. acknowledges funding from the Italian Association for Cancer Research (AIRC). R.H.H. was supported by a Rubicon fellowship from the Netherlands Organization for Scientific Research (NWO). The work in the Auwerx laboratory was supported by grants of the Ecole Polytechnique Fédérale de Lausanne, Swiss National Science Foundation, NIH (DK59820), the Velux Stiftung, and the European Research Council Ideas program (Sirtuins; ERC-2008-AdG23118). We thank all the members of the Auwerx laboratory for inspiring discussions.

\section{References}

Anido J, Saez-Borderias A, Gonzalez-Junca A, Rodon L, Folch G, Carmona MA, Prieto-Sanchez RM, Barba I, Martinez-Saez E, Prudkin L, et al. 2010. TGF- $\beta$ receptor inhibitors target the
CD44(high)/Id1(high) glioma-initiating cell population in human glioblastoma. Cancer Cell 18: 655-668.

Bao S, Wu Q, McLendon RE, Hao Y, Shi Q, Hjelmeland AB, Dewhirst MW, Bigner DD, Rich JN. 2006. Glioma stem cells promote radioresistance by preferential activation of the DNA damage response. Nature 444: 756-760.

Beier D, Hau P, Proescholdt M, Lohmeier A, Wischhusen J, Oefner PJ, Aigner L, Brawanski A, Bogdahn U, Beier CP. 2007. $\mathrm{CD}_{133^{+}}$and $\mathrm{CD}_{133^{-}}$glioblastoma-derived cancer stem cells show differential growth characteristics and molecular profiles. Cancer Res 67: 4010-4015.

Bolt MW, Mahoney PA. 1997. High-efficiency blotting of proteins of diverse sizes following sodium dodecyl sulfatepolyacrylamide gel electrophoresis. Anal Biochem 247: 185-192.

Boudoukha S, Cuvellier S, Polesskaya A. 2010. Role of the RNAbinding protein IMP-2 in muscle cell motility. Mol Cell Biol 30: $5710-5725$.

Calabrese C, Poppleton H, Kocak M, Hogg TL, Fuller C, Hamner B, Oh EY, Gaber MW, Finklestein D, Allen M, et al. 2007. A perivascular niche for brain tumor stem cells. Cancer Cell 11: 69-82.

Carroll J, Fearnley IM, Shannon RJ, Hirst J, Walker JE. 2003. Analysis of the subunit composition of complex I from bovine heart mitochondria. Mol Cell Proteomics 2: 117-126.

Carroll J, Fearnley IM, Skehel JM, Shannon RJ, Hirst J, Walker JE. 2006. Bovine complex I is a complex of 45 different subunits. J Biol Chem 281: 32724-32727.

Charles N, Ozawa T, Squatrito M, Bleau AM, Brennan CW, Hambardzumyan D, Holland EC. 2010. Perivascular nitric oxide activates notch signaling and promotes stem-like character in PDGF-induced glioma cells. Cell Stem Cell 6: 141-152.

Christiansen J, Kolte AM, Hansen TO, Nielsen FC. 2009. IGF2 mRNA-binding protein 2: Biological function and putative role in type 2 diabetes. J Mol Endocrinol 43: 187-195.

Clement V, Sanchez P, de Tribolet N, Radovanovic I, Ruiz i Altaba A. 2007. HEDGEHOG-GLI1 signaling regulates human glioma growth, cancer stem cell self-renewal, and tumorigenicity. Curr Biol 17: 165-172.

Clement V, Marino D, Cudalbu C, Hamou M-F, Mlynarik V, de Tribolet N, Dietrich P-Y, Gruetter R, Hegi ME, Radovanovic I. 2010. Marker-independent identification of glioma-initiating cells. Nat Methods 7: 224-228.

Clevers H. 2011. The cancer stem cell: Premises, promises and challenges. Nat Med 17: 313-319.

Currle DS, Gilbertson RJ. 2008. The niche revealed. Cell Stem Cell 3: 234-236.

Dai N, Rapley J, Angel M, Yanik MF, Blower MD, Avruch J. 2011. mTOR phosphorylates IMP2 to promote IGF2 mRNA translation by internal ribosomal entry. Genes Dev 25: 1159-1172.

Dean M, Fojo T, Bates S. 2005. Tumour stem cells and drug resistance. Nat Rev Cancer 5: 275-284.

Dimitriadis E, Trangas T, Milatos S, Foukas PG, Gioulbasanis I, Courtis N, Nielsen FC, Pandis N, Dafni U, Bardi G, et al. 2007. Expression of oncofetal RNA-binding protein CRD-BP/ IMP1 predicts clinical outcome in colon cancer. Int / Cancer 121: 486-494.

Ezhilarasan R, Mohanam I, Govindarajan K, Mohanam S. 2007. Glioma cells suppress hypoxia-induced endothelial cell apoptosis and promote angiogenic process. Int J Oncol 30: 701-707.

Fernandez-Vizarra E, Tiranti V, Zeviani M. 2009. Assembly of the oxidative phosphorylation system in humans: What we have learned by studying its defects. Biochim Biophys Acta 1793: 200-211.

Frank NY, Schatton T, Frank MH. 2010. The therapeutic promise of the cancer stem cell concept. J Clin Invest 120: 41-50. 
Galli R, Binda E, Orfanelli U, Cipelletti B, Gritti A, De Vitis S, Fiocco R, Foroni C, Dimeco F, Vescovi A. 2004. Isolation and characterization of tumorigenic, stem-like neural precursors from human glioblastoma. Cancer Res 64: 7011-7021.

Görlach A, Acker, H. 1994. pO2- and pH-gradients in multicellular spheroids and their relationship to cellular metabolism and radiation sensitivity of malignant human tumor cells. Biochim Biophys Acta 1227: 105-112.

Hammer NA, Hansen TO, Byskov AG, Rajpert-De Meyts E, Grondahl ML, Bredkjaer HE, Wewer UM, Christiansen J, Nielsen FC. 2005. Expression of IGF-II mRNA-binding proteins (IMPs) in gonads and testicular cancer. Reproduction 130: $203-212$.

Hanahan D, Weinberg RA. 2011. Hallmarks of cancer: The next generation. Cell 144: 646-674.

Himoto T, Kuriyama S, Zhang JY, Chan EK, Kimura Y, Masaki T, Uchida N, Nishioka M, Tan EM. 2005a. Analyses of autoantibodies against tumor-associated antigens in patients with hepatocellular carcinoma. Int J Oncol 27: 1079-1085.

Himoto T, Kuriyama S, Zhang JY, Chan EK, Nishioka M, Tan EM. 2005b. Significance of autoantibodies against insulinlike growth factor II mRNA-binding proteins in patients with hepatocellular carcinoma. Int J Oncol 26: 311-317.

Joo KM, Kim SY, Jin X, Song SY, Kong D-S, Lee JI, Jeon JW, Kim MH, Kang BG, Jung Y, et al. 2008. Clinical and biological implications of CD133-positive and CD133-negative cells in glioblastomas. Lab Invest 88: 808-815.

Jose C, Bellance N, Rossignol R. 2011. Choosing between glycolysis and oxidative phosphorylation: A tumor's dilemma? Biochim Biophys Acta 6: 552-561.

Kobel M, Weidensdorfer D, Reinke C, Lederer M, Schmitt WD, Zeng K, Thomssen C, Hauptmann S, Huttelmaier S. 2007. Expression of the RNA-binding protein IMP1 correlates with poor prognosis in ovarian carcinoma. Oncogene 26: 7584-7589.

Lapidot T, Sirard C, Vormoor J, Murdoch B, Hoang T, CaceresCortes J, Minden M, Paterson B, Caligiuri MA, Dick JE. 1994. A cell initiating human acute myeloid leukaemia after transplantation into SCID mice. Nature 367: 645-648.

Lee J, Kotliarova S, Kotliarov Y, Li A, Su Q, Donin NM, Pastorino S, Purow BW, Christopher N, Zhang W, et al. 2006. Tumor stem cells derived from glioblastomas cultured in bFGF and EGF more closely mirror the phenotype and genotype of primary tumors than do serum-cultured cell lines. Cancer Cell 9: 391-403.

Li Z, Bao S, Wu Q, Wang H, Eyler C, Sathornsumetee S, Shi Q, Cao Y, Lathia J, McLendon RE, et al. 2009. Hypoxia-inducible factors regulate tumorigenic capacity of glioma stem cells. Cancer Cell 15: 501-513.

Lottaz C, Beier D, Meyer K, Kumar P, Hermann A, Schwarz J, Junker M, Oefner PJ, Bogdahn U, Wischhusen J, et al. 2010. Transcriptional profiles of $\mathrm{CD}_{133^{+}}$and $\mathrm{CD} 133^{-}$glioblastomaderived cancer stem cell lines suggest different cells of origin. Cancer Res 70: 2030-2040.

Louis DN. 2006. Molecular pathology of malignant gliomas. Annu Rev Pathol 1: 97-117.

Magee JA, Piskounova E, Morrison SJ. 2012. Cancer stem cells: Impact, heterogeneity, and uncertainty. Cancer Cell 21: 283-296.

Mason MG, Nicholls P, Wilson MT, Cooper CE. 2006. Nitric oxide inhibition of respiration involves both competitive (heme) and noncompetitive (copper) binding to cytochrome c oxidase. Proc Nat Acad Sci 103: 708-713.

Murray J, Zhang B, Taylor SW, Oglesbee D, Fahy E, Marusich MF, Ghosh SS, Capaldi RA. 2003. The subunit composition of the human NADH dehydrogenase obtained by rapid onestep immunopurification. J Biol Chem 278: 13619-13622.
Nielsen J, Christiansen J, Lykke-Andersen J, Johnsen AH, Wewer UM, Nielsen FC. 1999. A family of insulin-like growth factor II mRNA-binding proteins represses translation in late development. Mol Cell Biol 19: 1262-1270.

Nijtmans LG, Henderson NS, Holt IJ. 2002. Blue Native electrophoresis to study mitochondrial and other protein complexes. Methods 26: 327-334.

Rehling P, Brandner K, Pfanner N. 2004. Mitochondrial import and the twin-pore translocase. Nat Rev Mol Cell Biol 5: 519530.

Reich M, Liefeld T, Gould J, Lerner J, Tamayo P, Mesirov JP. 2006. GenePattern 2.0. Nat Genet 38: 500-501.

Ricci-Vitiani L, Lombardi DG, Pilozzi E, Biffoni M, Todaro M, Peschle C, De Maria R. 2007. Identification and expansion of human colon-cancer-initiating cells. Nature 445: 111-115.

Ricci-Vitiani L, Pallini R, Biffoni $M$, Todaro $M$, Invernici $G$, Cenci T, Maira G, Parati EA, Stassi G, Larocca LM, et al. 2010. Tumour vascularization via endothelial differentiation of glioblastoma stem-like cells. Nature 468: 824-828.

Rong Y, Durden DL, Van Meir EG, Brat DJ. 2006. 'Pseudopalisading' necrosis in glioblastoma: A familiar morphologic feature that links vascular pathology, hypoxia, and angiogenesis. J Neuropathol Exp Neurol 65: 529-539.

Saxena R, Voight BF, Lyssenko V, Burtt NP, de Bakker PI, Chen H, Roix JJ, Kathiresan S, Hirschhorn JN, Daly MJ, et al. 2007. Genome-wide association analysis identifies loci for type 2 diabetes and triglyceride levels. Science 316: 1331-1336.

Scott LJ, Mohlke KL, Bonnycastle LL, Willer CJ, Li Y, Duren WL, Erdos MR, Stringham HM, Chines PS, Jackson AU, et al. 2007. A genome-wide association study of type 2 diabetes in Finns detects multiple susceptibility variants. Science 316: 1341-1345.

Singh SK, Hawkins C, Clarke ID, Squire JA, Bayani J, Hide T, Henkelman RM, Cusimano MD, Dirks PB. 2004. Identification of human brain tumour initiating cells. Nature 432: 396-401.

Son MJ, Woolard K, Nam D-H, Lee J, Fine HA. 2009. SSEA-1 is an enrichment marker for tumor-initiating cells in human glioblastoma. Cell Stem Cell 4: 440-452.

Suissa M, Schatz G. 1982. Import of proteins into mitochondria. Translatable mRNAs for imported mitochondrial proteins are present in free as well as mitochondria-bound cytoplasmic polysomes. I Biol Chem 257: 13048-13055.

Sun L, Hui A-M, Su Q, Vortmeyer A, Kotliarov Y, Pastorino S, Passaniti A, Menon J, Walling J, Bailey R, et al. 2006. Neuronal and glioma-derived stem cell factor induces angiogenesis within the brain. Cancer Cell 9: 287-300.

Sutherland RM. 1998. Tumor hypoxia and gene expressionimplications for malignant progression and therapy. Acta Oncol 37: 567-574.

Suvà M-L, Riggi N, Janiszewska M, Radovanovic I, Provero $\mathrm{P}$, Stehle J-C, Baumer K, Le Bitoux M-A, Marino D, Cironi L, et al. 2009. EZH2 is essential for glioblastoma cancer stem cell maintenance. Cancer Res 69: 9211-9218.

Sylvestre J, Margeot A, Jacq C, Dujardin G, Corral-Debrinski M. 2003. The role of the $3^{\prime}$ untranslated region in mRNA sorting to the vicinity of mitochondria is conserved from yeast to human cells. Mol Biol Cell 14: 3848-3856.

Vander Heiden MG, Cantley LC, Thompson CB. 2009. Understanding the Warburg effect: The metabolic requirements of cell proliferation. Science 324: 1029-1033.

Vaupel P, Kallinowski F, Okunieff P. 1989. Blood flow, oxygen and nutrient supply, and metabolic microenvironment of human tumors: A review. Cancer Res 49: 6449-6465.

Verhaak RGW, Hoadley KA, Purdom E, Wang V, Qi Y, Wilkerson MD, Miller CR, Ding L, Golub T, Mesirov JP, et al. 2010. 
Janiszewska et al.

Integrated genomic analysis identifies clinically relevant subtypes of glioblastoma characterized by abnormalities in PDGFRA, IDH1, EGFR, and NF1. Cancer Cell 17: 98-110.

Vlashi E, Lagadec C, Vergnes L, Matsutani T, Masui K, Poulou M, Popescu R, Della Donna L, Evers P, Dekmezian C, et al. 2011. Metabolic state of glioma stem cells and nontumorigenic cells. Proc Natl Acad Sci 108: 16062-16067.

Vogel RO, Smeitink JAM, Nijtmans LGJ. 2007. Human mitochondrial complex I assembly: A dynamic and versatile process. Biochim Biophys Acta 1767: 1215-1227.

Wakimoto H, Kesari S, Farrell CJ, Curry WT, Zaupa C, Aghi M, Kuroda T, Stemmer-Rachamimov A, Shah K, Liu T-C, et al. 2009. Human glioblastoma-derived cancer stem cells: Establishment of invasive glioma models and treatment with oncolytic herpes simplex virus vectors. Cancer Res 69: 3472-3481.

Wakimoto H, Mohapatra G, Kanai R, Curry WT, Yip S, Nitta M, Patel AP, Barnard ZR, Stemmer-Rachamimov AO, Louis DN, et al. 2012. Maintenance of primary tumor phenotype and genotype in glioblastoma stem cells. Neuro-oncol 14: 132-144.

Wang R, Chadalavada K, Wilshire J, Kowalik U, Hovinga KE, Geber A, Fligelman B, Leversha M, Brennan C, Tabar V. 2010. Glioblastoma stem-like cells give rise to tumour endothelium. Nature 468: 829-833.

Yaniv K, Yisraeli JK. 2002. The involvement of a conserved family of RNA binding proteins in embryonic development and carcinogenesis. Gene 287: 49-54.

Zoncu R, Efeyan A, Sabatini DM. 2011. mTOR: From growth signal integration to cancer, diabetes and ageing. Nat Rev Mol Cell Biol 12: 21-35. 


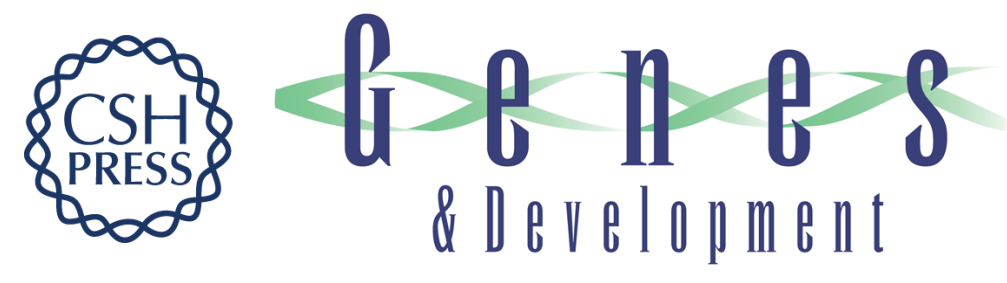

\section{Imp2 controls oxidative phosphorylation and is crucial for preserving glioblastoma cancer stem cells}

Michalina Janiszewska, Mario L. Suvà, Nicolo Riggi, et al.

Genes Dev. 2012, 26: originally published online August 16, 2012

Access the most recent version at doi:10.1101/gad.188292.112

\section{Supplemental http://genesdev.cshlp.org/content/suppl/2012/08/09/gad.188292.112.DC1 Material}

References This article cites 63 articles, 21 of which can be accessed free at: http://genesdev.cshlp.org/content/26/17/1926.full.html\#ref-list-1

\section{License}

Email Alerting

Receive free email alerts when new articles cite this article - sign up in the box at the top Service

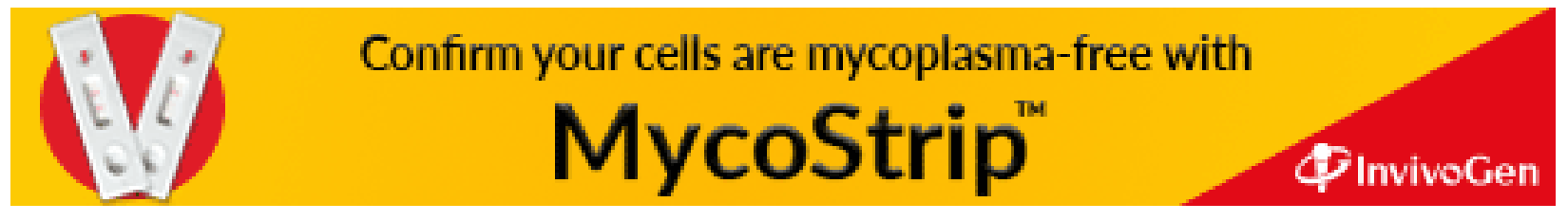

